\title{
Thermal history and structure of rotating protoneutron stars with relativistic equation of state
}

\author{
K. Sumiyoshi ${ }^{1}$, J.Ma Ibáñez $^{2}$, and J.V. Romero ${ }^{3}$ \\ 1 The Institute of Physical and Chemical Research, (RIKEN), Wako, Saitama 351-01, Japan \\ 2 Departamento de Astronomía y Astrofísica, Universidad de Valencia, 46100 Burjassot, Spain \\ 3 Departamento de Matemática Aplicada, Universidad Politécnica de Valencia, 46071 Valencia, Spain
}

Received March 17; accepted July 7, 1998

\begin{abstract}
We study the properties of general relativistic, slowly rotating, protoneutron stars. We explore the structure of rotating protoneutron stars with a wide range of the entropy per baryon, the lepton fraction and the baryonic mass in order to study the evolutionary history of protoneutron stars during the cooling epoch. We adopt the relativistic equation of state for dense matter derived within the relativistic mean field theory, which is based on the microscopic nuclear many-body framework, and checked by the experimental data of many nuclei. We provide broad information on the effect of rotation, temperature and lepton trapping of protoneutron stars with various baryonic masses. The evolutionary track of rotating protoneutron stars is examined and its implications are addressed.
\end{abstract}

Key words: dense matter — stars: interiors — stars: neutron - stars: rotation

\section{Introduction}

A protoneutron star is a very hot $(T \leq 50-60 \mathrm{MeV})$ and lepton rich object in quasi-hydrostatic equilibrium (Burrows \& Lattimer 1986). These stars are formed in the gravitational collapse of iron cores in massive stars (for alternative scenarios on the origin of neutron stars see, e.g., the review by Canal et al. 1990). During the first $25-30$ s. in the life of the protoneutron star - the Kelvin epoch for neutrino cooling or the core deleptonization era - the temperature in the core decreases to a typical central value of $\sim 8 \mathrm{MeV}$, with a profile (in radius) like that given by the relativistic thermal equilibrium condition (see Burrows 1990 for a review on this subject).

Send offprint requests to: J.V. Romero

Correspondence to: Jose.Romero@uv.es
Recent two-dimensional hydrodynamical calculations show that convection in the protoneutron star can encompass the whole star within $\approx 1 \mathrm{~s}$, and in this epoch a near constant profile in entropy is observed, with a typical value of $s=3$ (Janka \& Müller 1996; Keil et al. 1996). The extension of the convective region in a protoneutron star is a matter of debate. Many independent models must prove that protoneutron stars have long-lasting convection, before this can be considered as an established fact.

On the other hand, detailed studies of the evolution of massive stars carried out by Woosley and collaborators lead to the conclusion that massive iron cores up to $\approx 2.15 M_{\odot}$ (Model $2.0 \mathrm{~S}$ of $40 M_{\odot}$ of the the review of evolutionary calculations, without mass loss, by Weaver \& Woosley 1993), can be formed during the late stages of their evolution. In Woosley et al. (1993) was reported for the first time the complete evolution of a set of massive stars, from the main sequence to the presupernova stage, including mass loss, and the authors found models which develop iron cores of up to $\approx 1.9 M_{\odot}$ (presupernova model 85WRA of $\left.85 M_{\odot}\right)$.

Consequently, it would be interesting to take these facts into account in order to clarify the conditions of the interior of a protoneutron star: isentropic or isothermal (see, Goussard et al. 1997, for a discussion) and its evolutionary history.

The study of the properties of such protoneutron stars is important to understand supernova neutrinos emitted during the cooling epoch (Burrows \& Lattimer 1986; Suzuki 1994). The characteristics of a neutrino burst reflect the thermal condition inside the star and its cooling history. The profile of a neutrino burst may also affect the mechanism of the supernova explosion itself (See Janka \& Müller 1996, for example). Axisymmetric neutrino emission from rotating protoneutron stars have been of particular interest. The effect this may have on the observation of supernova neutrinos has been pointed out (Janka \& Mönchmeyer 1989) and a mechanism has been 
proposed for supernova explosion through anisotropic neutrino heating (Shimizu et al. 1994). To discuss these effects it is essential to know the size of the deformation and the density profile (density gradient, for example), which determine the flux of neutrinos, of rotating protoneutron stars. Therefore, it is of great interest to investigate the structure of rotating protoneutron stars taking into account the thermal profile and history.

After the seminal paper by Burrows \& Lattimer (1986) several authors have studied the properties of static non-rotating protoneutron stars. Let us cite some of them: Sumiyoshi \& Toki (1994); Takatsuka (1995); Bombaci et al. (1995); Sumiyoshi et al. (1995a,c); Bombaci (1996); Bombaci et al. (1996) and the ones contained in Glendenning's book (Glendenning 1997).

A few papers have appeared recently which focus on the analysis of the stability of rapidly rotating protoneutron stars (Hashimoto et al. 1994; Goussard et al. 1997) using a full general-relativistic description of axisymmetric configurations. The main differences between these calculations refer to the assumptions concerning: i) the thermal state of the hot interior of the protoneutron star - isothermal (according to the Newtonian definition in Hashimoto et al. 1995, or the Einsteinian definition in Goussard et al. 1997), or isentropic (in the case of Goussard et al. 1997) -, and ii) the lepton composition, $Y_{\mathrm{L}}$, is zero in Hashimoto et al. (1994), but Goussard et al. (1997) consider two extreme values $Y_{\mathrm{L}}=0,0.4$ in their calculations. The EOS adopted in these studies are different and several sets of EOSs which differ in stiffness are used in Hashimoto et al. (1994) whereas Goussard et al. (1997) use a set of EOS by Lattimer \& Swesty (1991).

It should be stressed that the computations carried out by Goussard et al. (1997; see also Goussard 1997 for details) are careful as they combine the following: i) high accuracy of spectral methods, ii) checking their results with the general-relativistic virial theorem, and iii) a thermodynamically consistent interpolation in the EOS tables. A main conclusion coming from the calculations made by Goussard et al. (1997) is the existence of a minimum rotation period of rapidly rotating protoneutron stars which is significantly larger than the corresponding limit for cold neutron stars. For the range of gravitational masses $1.3-1.8 M_{\odot}$, Goussard et al. (1997) obtain minimum rotation periods of $1.25-1.1 \mathrm{~ms}(1.6-1.1 \mathrm{~ms})$, respectively, for their isothermal and deleptonized (trapped lepton, $Y_{\mathrm{L}}=0.4$, and isentropic) models. Hence, a fundamental lower limit of $1.1 \mathrm{~ms}$ seems to arise from these calculations (Hashimoto et al. 1994, obtain a lower limit of $0.9 \mathrm{~ms})$.

The rotation of a star is limited by the Kepler frequency, $\Omega_{\mathrm{k}}$, at which the equatorial surface velocity equals the orbital velocity of a particle at the equator and, hence, mass shedding at the stellar equator makes the star unstable. If $M_{\max }$ and $R_{\max }$ are the gravitational mass and the radius, respectively, of the non-rotating equilibrium model at the maximum of the corresponding curve for a given equation of state (EOS), the following empirical formula has been proved to be a reasonable approximation of the limiting frequency

$\Omega_{\mathrm{k}}=C\left(M_{\max } / M_{\odot}\right)^{1 / 2}\left(R_{\max } / 10 \mathrm{~km}\right)^{-3 / 2}$,

where parameter $C$ can have some of the following values: i) $C_{\mathrm{N}} \approx 1.1510^{4} \mathrm{~s}^{-1}$ in the Newtonian case, ii) $C_{\mathrm{HZ}} \approx 0.77510^{4} \mathrm{~s}^{-1}$ according to the fitting, derived by Haensel \& Zdunik (1989), to the full (axisymmetric) general relativistic results of Friedman et al. $(1986,1989)$ and iii) $C_{\mathrm{G}} \approx(0.750-0.830) 10^{4} \mathrm{~s}^{-1}$ according to the calculations carried out by Goussard et al. (1997; see also Goussard 1997), the general trend being that $C_{\mathrm{G}}$ increases with the central temperature (isothermal models), decreases with entropy (isentropic models) and increases with the incompressibility modulus.

If we define the adimensional parameter $\eta \equiv \Omega / \Omega_{\mathrm{k}}$ where $\Omega$ is the angular velocity of the star as measured by a distant observer (we will assume that $\Omega$ is constant inside the star, i.e., the star rotates uniformly) and consider the Newtonian limit of $\Omega_{\mathrm{k}}$, then two values of $\eta$ are of particular relevance: i) $\eta \approx 0.7$, for a star having the limit frequency given by the above Haensel \& Zdunik fitting, and ii) $\eta \approx 0.3$ which is the approximate value for a millisecond pulsar (like, e.g., PSR 1937+21).

Consequently, with the above estimate the assumption of slow rotation following Hartle's approach (1967) seems to be a good way to look more deeply into the properties of rotating relativistic equilibrium configurations. This approach combines: i) simplicity, ii) it is sufficient to understand the main macroscopic properties of most pulsars (with exception, perhaps, of the millisecond ones), and iii) it enables one to know the influence of general relativistic effects on the pulse profile (Kapoor \& Datta 1985, 1986; Datta 1988). Therefore, consistent with Hartle's approach, we will assume that the star rotates like a solid rigid object, in spite of the fact that, in a more realistic scenario, rotation is differential (Janka \& Mönchmeyer 1989).

Unlike the paper by Goussard et al. (1997) we have made an exhaustive analysis of the properties of slowly rotating protoneutron stars, and focussed on the dependence of these properties on the two thermodynamical parameters which we consider constant inside the star: $Y_{\mathrm{L}}$ (the lepton fraction) and $s$ (the entropy per baryon). The assumption of a constant profile for $Y_{\mathrm{L}}$ is realistic, once $\beta$-equilibrium conditions have been reached inside the inner core, during the infall epoch. We have also paid particular attention to the evolutionary track of cooling protoneutron stars by exploring a wide range of values for the thermodynamical parameters as well as the baryonic mass of the star. The assumption of a constant profile for $s$ is based on the considerations above regarding convection in protoneutron stars.

Another aim of the current study is to explore the properties of protoneutron stars adopting the 
relativistic EOS, which is qualitatively different from conventional EOSs (Sumiyoshi et al. 1995). The recent success of relativistic nuclear many-body frameworks (see Serot \& Walecka 1986, for a review) is of great importance for astrophysical applications. In particular, the relativistic mean field (RMF) theory has been quite successful in describing the EOS as well as the properties of the nuclei. It should be stressed that the RMF theory has been extensively checked by experimental data of nuclei in the region away from stability, where the environment is closer to neutron stars, and thus the EOS is well constrained by the current knowledge of nuclear physics (Hirata et al. 1991; Sugahara \& Toki 1994; Hirata et al. 1997).

In Sect. 2 we describe the relativistic EOS used in the present paper. Section 3 discusses the properties of the slowly rotating protoneutron stars coming from our calculation. Section 4 shows some evolutionary sequences at fixed baryon mass and angular momentum in a diagram entropy vs. central rest-mass density. Conclusions are summarized in Sect. 5.

\section{Relativistic EOS for dense matter at finite temperature}

We adopt the relativistic mean field (RMF) theory to derive all the physical quantities of dense matter at finite temperature. The RMF theory is one of nuclear manybody frameworks based on an effective Lagrangian, which is relativistically covariant, composed of nucleon and meson degrees of freedom. We refer to the review article by Serot \& Walecka (1986) for details concerning with the relativistic many-body framework. All the details on the relativistic EOS for neutron stars and supernovae in the RMF theory can be found in recent papers (Sumiyoshi \& Toki 1994; Sumiyoshi et al. 1995).

We start with the phenomenological Lagrangian composed of nucleon field and $\sigma, \omega$ and $\rho$ meson fields and solve the relativistic many-body problem within the mean field approximation. The adopted Lagrangian also contains non-linear $\sigma$ and $\omega$ meson terms (Sugahara \& Toki 1994), which are motivated by the recent success of the relativistic Brückner Hartree Fock (RBHF) theory (Brockmann \& Machleidt 1990). It should be noted that the RBHF theory can reproduce the saturation property of nuclear matter starting from the elementary nucleonnucleon interaction. RMF has been used very successfully as an effective theory of RBHF to describe dense matter and nuclear structure (Sugahara \& Toki 1994).

The Lagrangian contains the parameters: $\sigma$ meson mass, coupling constants between nucleon and mesons and self-coupling constants of mesons. We adopt the best parameter set called TMA, which has been determined by fitting the experimental data of masses and radii of many nuclei in the periodic table (Sugahara 1995; Hirata et al. 1997). The symmetry energy of EOS using TMA is
$30.68 \mathrm{MeV}$ and the incompressibility is $318 \mathrm{MeV}$. The RMF theory with TMA has been applied to studying the ground state properties of about 2000 even-even nuclei covering all the region of the nuclear chart. It has also proved very successful in reproducing the experimental data of stable nuclei as well as unstable nuclei (Hirata et al. 1997). The prediction of nuclear structure in the RMF theory with TMA is being checked in experiments on radioactive nuclear beam facilities in the world, so we can constrain the EOS for astrophysics at the same time.

The extension of the RMF theory to finite temperature is straightforward (Serot \& Walecka 1986; Sumiyoshi \& Toki 1994). Another field theoretical approach to provide EOS at finite temperature has been worked out (Diaz Alonso et al. 1989) and has been applied to studying warm cores in neutron stars (Martí et al. 1988; Romero et al. 1992). All the physical quantities of dense matter under various conditions of chemical composition, density and temperature are calculated in the RMF theory with TMA and are prepared in the form of a table of numerical data, which is available for numerical simulations of protoneutron star cooling and supernova explosion (Sumiyoshi \& Toki 1994).

In the present study, we assume that the dense matter in protoneutron stars is composed of neutrons, protons, electrons, neutrinos, their anti-particles and photons, and satisfies the chemical equilibrium and the charge neutrality. We characterize the dense matter by the lepton fraction $Y_{\mathrm{L}}$ and the entropy per baryon $s$, which we assume to be constant inside stars. We calculate the EOS of the dense matter under the above condition by adding lepton contributions to nuclear contributions and use them to calculate the equilibrium configuration of slowly rotating protoneutron stars. As for the EOS of dense matter at low densities below $10^{14} \mathrm{~g} / \mathrm{cm}^{3}$, we adopt the EOS by Lattimer \& Swesty (1991) with the addition of lepton contributions and the constraints on $Y_{\mathrm{L}}$ and $s$ and connect smoothly with the EOS by the RMF theory with TMA at high density.

The properties of the relativistic EOS in the RMF theory and the profiles of non-rotating neutron stars and protoneutron stars using the relativistic EOS have been reported in detail by Sumiyoshi \& Toki (1994) and Sumiyoshi et al. (1995a). It is to be noted here that the relativistic EOS has different characteristics compared to the conventional EOS in non-relativistic frameworks. For example, the proton fraction inside the neutron stars using the relativistic EOS is remarkably large (see also Sumiyoshi et al. 1995b). The current study is the first result of the application of relativistic EOS, taking into account the conditions on $Y_{\mathrm{L}}$ and $s$, in the RMF theory to rotating protoneutron stars. It would be of great interest to explore the characteristics of rotating protoneutron stars by comparing them with nonrotating ones and other studies with conventional EOSs. 

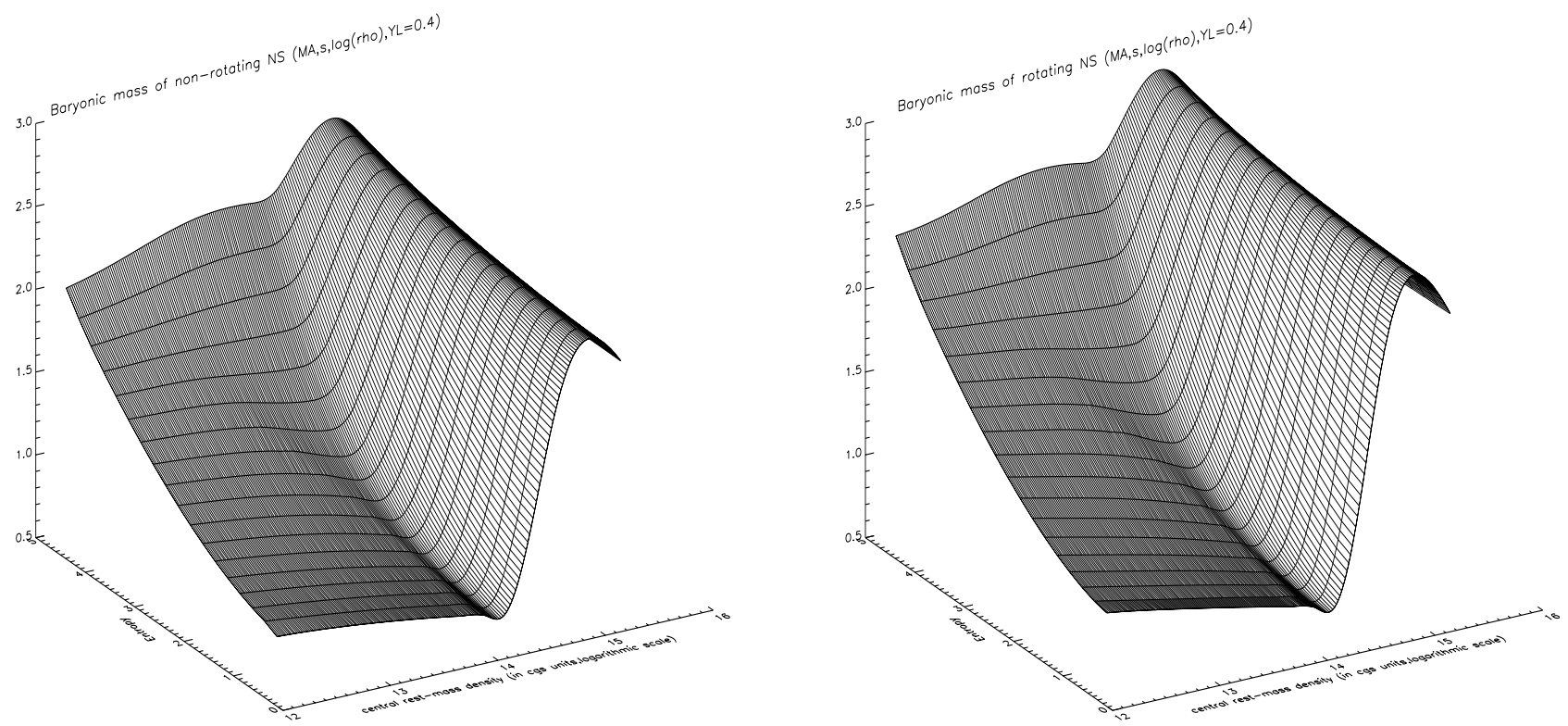

Fig. 1. Baryonic mass (in solar units) as a function of the central rest-mass density (in cgs units and logarithmic scale) and entropy (in units of the Boltzmann constant) for the non-rotating equilibrium configurations (left) and for rotating configurations (right)

\section{Properties of slowly rotating protoneutron stars}

We have calculated the equilibrium configurations of slowly rotating protoneutron stars according to the above EOS in the framework of the Einsteinian theory of gravitation. This task has been done by solving the equations derived by Hartle numerically (Hartle 1967; Hartle \& Thorne 1968 ) in order to describe the structure of slowly rotating relativistic stars (see Appendix for a summary). Hartle's formalism, basically, consists in perturbing the geometry of spacetime around a static and spherical background. Although Hartle's approach was developed for cold EOS, its generalization to isentropic configurations is straightforward.

Tables 1 and 2 summarize the main macroscopic properties of the protoneutron stars for $Y_{\mathrm{L}}=0.3,0.4$ respectively. The table headings show the different magnitudes (see below, and also the Appendix, for definitions) allowing for configuration characterisation. For each value of the entropy, we have selected three models: the one corresponding to the minimum of gravitational mass, the standard $1.4 M_{\odot}$, and the maximum of gravitational mass, $M_{\max }$.

Let us note that the value of $\Omega$ has been normalized to the Newtonian critical one $\left(C=C_{\mathrm{N}}\right.$ in Eq. (1.1)) in the figures shown in this paper and to the Haensel and Zdunik fitting ( $C=C_{\mathrm{HZ}}$ in Eq. (1.1)) in the results shown in Tables 1 and 2 . Hence, there is an overestimation of rotation effects (at the limit where the formalism breaks down) but due to the well-known scaling laws with $\Omega$ satisfied by the different magnitudes, it is easy to extend our results to any lower $\Omega$ value (see Appendix). Glendenning
(1997, and references therein) has reported that the selfconsistency condition for the keplerian angular velocity in Hartle's perturbative approach satisfies the following useful approximation:

$\Omega_{\mathrm{k}}=\left(1+\frac{\omega(R)}{\Omega_{\mathrm{k}}}-\left(\frac{\omega(R)}{\Omega_{\mathrm{k}}}\right)^{2}\right)^{-1 / 2}\left(\frac{M}{R^{3}}\right)^{1 / 2}$

(see Appendix for definition of $\omega(r))$. Typical values of $\frac{\omega(R)}{\Omega_{\mathrm{k}}}$ are $\leq 0.8$, hence Glendenning's considerations lead to a critical value of the rotational frequency which can be fitted by a formula like Eq. (1.1) with a value of $C=C_{\mathrm{Gl}}$ a few percent lower than the Newtonian one $\left(C_{\mathrm{Gl}} \approx 0.9 C_{\mathrm{N}}\right)$.

Figure 1 shows the surfaces spanned by the baryonic mass of the equilibrium configurations for $Y_{\mathrm{L}}=0.4$ as a function of the entropy and the central rest-mass density, $M_{\mathrm{A}}=M_{\mathrm{A}}\left(s, \rho_{\mathrm{c}}\right)$, for the non-rotating (left) and rotating protoneutron stars (right). They summarize the main features of $M_{\mathrm{A}}$ : i) increasing function with $s$ and ii) existence of a minimum and a maximum for $M_{\mathrm{A}}$ with $\rho_{\mathrm{c}}$ which bind the set of stable configurations. Qualitatively similar results are obtained for $Y_{\mathrm{L}}=0.3$.

In Fig. 2 we have plotted the baryonic mass (left) and the gravitational mass (right) as a function of radius, for a selection of two extreme values of $s, s=0.8,5$. Both the nonrotating and rotating sequences of equilibrium configurations have been displayed for comparison. Curves corresponding to the same entropy value (indicated by labels) have been drawn with the same line type and different thickness (thicker for the rotating configurations and thinner for the non-rotating ones). Concerning this figure, we would like to point out the following: thermal effects have a stronger influence on the radius of the equilibrium 

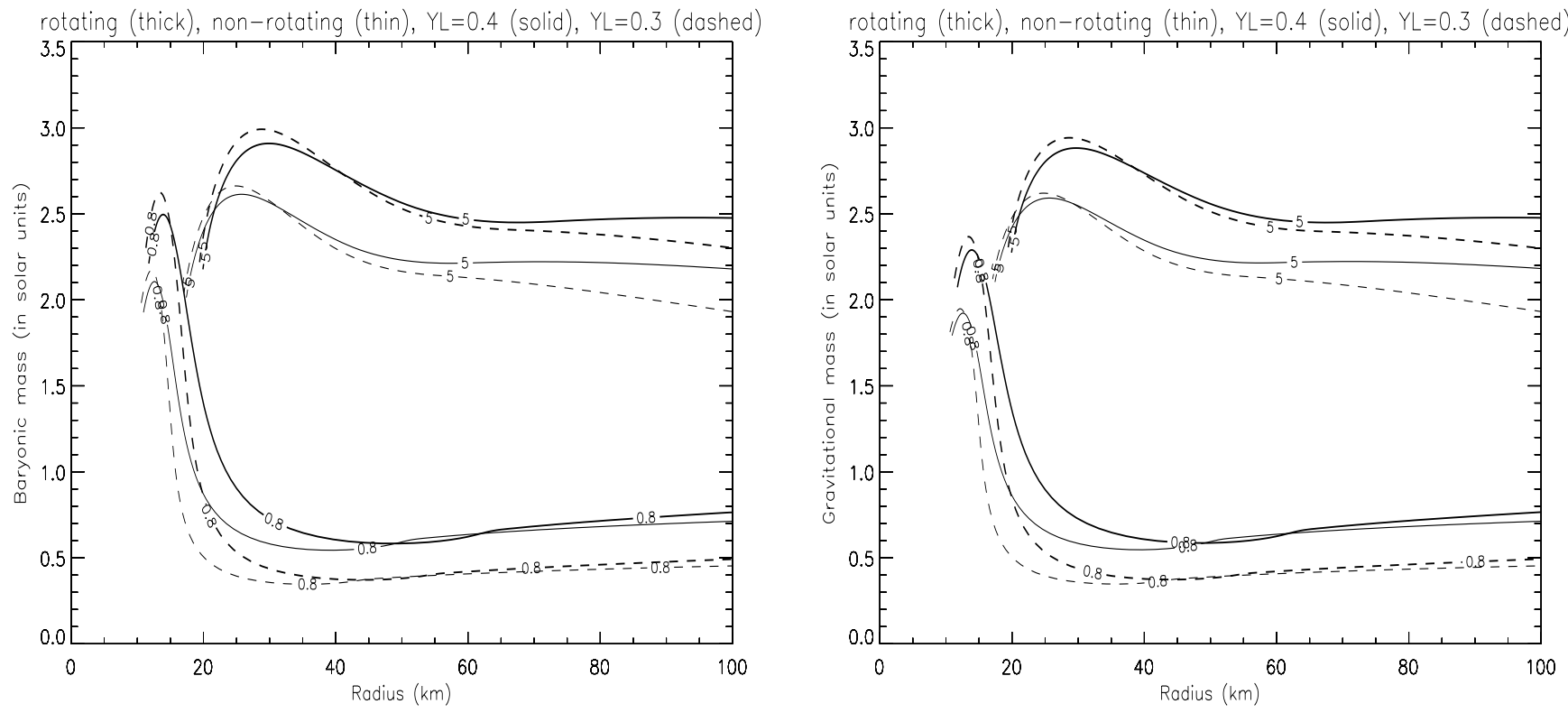

Fig. 2. Baryonic (left) and Gravitational mass (right), in solar units, as a function of the radius (in km) for two extreme values of entropy (labels stand for values $s=0.8$ and 5, in units of the Boltzmann constant) for the non-rotating (thin) and rotating (thick) equilibrium configurations. Solid (dashed) lines correspond to $Y_{\mathrm{L}}=0.4\left(Y_{\mathrm{L}}=0.3\right)$

configurations than those caused by rotation. The radius is the most sensitive to thermal effects since the atmosphere of the protoneutron star is placed at low densities and its radius increases for weak gravitational fields. At these low densities thermal pressure dominates over other non-thermal sources of pressure.

If we consider a rotating model with a canonical mass of $1.4 M_{\odot}$, its radius (see Eq. (A7) in the Appendix) satisfies: i) for $Y_{\mathrm{L}}=0.3$ is $14.6 \leq R(\mathrm{~km}) \leq 150$ when $0.75 \leq s \leq 4.5$ (see Table 1), and ii) for $Y_{\mathrm{L}}=0.4$ is $16.0 \leq R(\mathrm{~km}) \leq 40.1$ when $0.75 \leq s \leq 3.75$ (see Table 2). On the other hand, maximum mass depends less on temperature than radius: i) for $Y_{\mathrm{L}}=0.3$ the maximum gravitational mass is $1.95 \leq M / M_{\odot} \leq 2.62$ for $0.75 \leq s \leq 5.0$ (see Table 1), and ii) for $Y_{\mathrm{L}}=0.4$ it is $1.92 \leq M / M_{\odot} \leq 2.59$ for $0.75 \leq s \leq 5.0$ (see Table 2).

As can be seen in Tables 1 and 2 and Fig. 2, the gravitational mass of the rotating models increases up to $\approx 12 \%$ relative to the nonrotating ones. The correction to the gravitational mass, $\delta M$, is greater in configurations of intermediate mass and for low entropy values. From Tables 1 and 2 , we can verify the following relationship for $0.75 \leq s: \delta M / M \leq 0.12$ and $\delta M / M \leq 0.09$ for $1.4 M_{\odot}$ and $M_{\max }$, respectively. Corrections to the radius induced by rotation, $\delta R$, are more significant for models with intermediate masses than for those having the maximum mass at any entropy value. For example, the correction for $0.75 \leq s \leq 4.5$ satisfies the relation, $0.05 \leq \delta R / R \leq 0.10$ and $0.03 \leq \delta R / R \leq 0.05$ for $1.4 M_{\odot}$ and $M_{\max }$, respectively, as can be seen in Tables 1 and 2 .

Binding energy (Fig. 3, left) and gravitational mass (Fig. 3, right) as a function of central rest-mass density have been plotted for two values of $Y_{\mathrm{L}}(0.4$ and 0.3$)$ and for two extreme entropy values ( 0.8 and 5 ) by comparing nonrotating and rotating configurations. The corrections on binding energy, $\delta E_{\mathrm{B}}$, induced by rotation are consistent with the fact that the most bounded configurations have the most important corrections. When thermal effects are included, less bounded configurations are obtained (see Fig. 3, left) and the importance of the corrections induced by rotation decreases with entropy. Hence, e.g., quantity $\delta E_{\mathrm{B}} / M_{\odot}$ of the $1.4 M_{\odot}$ decreases from $1.510^{-2}$ to $1.710^{-4}$ when entropy increases in the interval $0.75 \leq s \leq$ 4.5 for $Y_{\mathrm{L}}=0.3$ (see Tables 1 and 2).

The quantity $\bar{\omega} \equiv \Omega-\omega$ is the angular velocity of the fluid relative to the local inertial frame (LIF). It measures the dragging of inertial frames. In this expression $\omega$, the angular velocity of the LIF, is proportional to the star's angular velocity $\Omega$ and, physically, stands for the angular velocity of a freely falling particle. In other words, LIFs are frames with rotational angular velocities $\omega(r)$ as measured by an observer at infinity at rest with respect to the star. On the surface, quantity $\bar{\omega}(R)$ is the effective angular velocity of a mass element at the star's surface and, in some sense, it determines the strength of centrifugal forces from a Newtonian point of view (Glendenning 1997). The function $w_{1} \equiv \bar{\omega} / \Omega$, evaluated at the surface, is shown in Fig. 4 (for $Y_{\mathrm{L}}=0.4$ and 0.3 ) in terms of the central rest-mass density. From Fig. 4 and Tables 1 and 2, several features can be addressed: frame dragging varies inversely to the star radius, it increases with central rest-mass density up to $\approx 20 \%$, and decreases with entropy.

The moment of inertia (see Appendix \& Hartle 1967, for definition) is shown, as a function of the central 
Table 1. Macroscopic properties of warm rotating neutron stars $\left(Y_{1}=0.3\right)$

\begin{tabular}{|c|c|c|c|c|c|c|c|c|c|c|c|c|}
\hline$S\left(K_{\mathrm{B}}\right)$ & $\rho_{14}$ & $M / M_{\odot}$ & $R(\mathrm{~km})$ & $E_{\mathrm{B}} / M_{\odot}$ & $I_{44}$ & $\Omega_{\mathrm{c}}\left(\mathrm{s}^{-1}\right)$ & $\omega_{1}$ & $\delta R / R$ & $M / M$ & ${ }^{e} \mathrm{~S}$ & $/ M R^{2}$ & $\delta E_{\mathrm{B}} / M_{\odot}$ \\
\hline \multirow[t]{3}{*}{$7.50 E-1$} & & & & & & & & & & & & \\
\hline & & & +1 & & & & & & -16 & & & . \\
\hline & $E+1$ & $5 E+0$ & $E+1$ & & & & & & & & & \\
\hline \multirow{3}{*}{$1.00 E+0$} & $E+0$ & $E-1$ & $3.72 E+1$ & -1 & $7.05 E+0$ & $6.47 E+2$ & 9 & & & & & \\
\hline & $6.71 E+0$ & $1.40 E+0$ & $1.49 E+1$ & $8.74 E-2$ & $1.78 E+1$ & $4.97 E+3$ & $9.22 E-1$ & $5.53 E-2$ & $1.18 E-1$ & $6.62 E-1$ & $3.43 E$ & \\
\hline & & 0 & $1.23 E+1$ & & & & & & & & & \\
\hline \multirow[t]{3}{*}{$1.25 E+0$} & 1.9 & 3.8 & $3.78 E+1$ & -1 . & $E+0$ & +2 & & & & 6 . & & \\
\hline & $6.43 E+0$ & & $1.54 E+1$ & & & & & & & $6.62 E$ & & \\
\hline & & & & & & & & & & & & \\
\hline \multirow[t]{3}{*}{$1.50 E+0$} & 1.7 & 4.1 & +1 & -1 . & +0 & $E+2$ & -1 & & & & & \\
\hline & 6.11 & & & & & & & & & & & \\
\hline & & & & & & & & & & & & \\
\hline \multirow[t]{3}{*}{$1.75 E+0$} & 1.7 & & 4.0 & -1 & +1 & +2 & & & & & & \\
\hline & 5.82 & & & & & & & & & & & \\
\hline & & & & & & & & & & & & \\
\hline \multirow[t]{3}{*}{$2.00 E+0$} & 1.58 & & 4.3 & -2. & 1.3 & +2 & 9.9 & & & & & \\
\hline & & & & & & & & & & & & \\
\hline & & & & & & & & & & & & \\
\hline \multirow[t]{3}{*}{$2.25 E+0$} & 1.52 & & 4.38 & -2 & 1.6 & +2 & & & & & & \\
\hline & & & & & & & & & & & & \\
\hline & & & & & & & & & & & & \\
\hline \multirow[t]{3}{*}{$2.50 E+0$} & 1.35 & & 4.7 & -2. & & & & & & & & \\
\hline & & & & & & & & & & & & \\
\hline & & & & & & & & & & & & \\
\hline \multirow[t]{3}{*}{$2.75 E+0$} & $1.25 E+0$ & 1 & 4.9 & -2 . & 2.7 & +2 & & & & & & \\
\hline & & & & & & & & & & & & \\
\hline & & & & & & & & & & & & \\
\hline \multirow[t]{3}{*}{$3.00 E+0$} & & & & & & & & & & & & \\
\hline & & & & & & & & & & & & \\
\hline & & & & & & & & & & & & \\
\hline \multirow[t]{3}{*}{$3.25 E+0$} & 9.4 & & & & & & & & & & & \\
\hline & & & & & & & & & & & & \\
\hline & & & & & & & & & & & & \\
\hline \multirow[t]{3}{*}{$3.50 E+0$} & & & & & & & & & & & & \\
\hline & & & & & & & & & & & & \\
\hline & & & & & & & & & & & & \\
\hline \multirow[t]{3}{*}{$3.75 E+0$} & & & & -2 & & & & & & & & \\
\hline & & & & & & & & & & & & \\
\hline & & & & & & & & & & & & \\
\hline \multirow[t]{2}{*}{$4.00 E+0$} & & & & & & & & & & & & \\
\hline & & & & & & & & & & & & \\
\hline \multirow{2}{*}{$4.25 E+0$} & & & & & & & & & & & & \\
\hline & & & & & & & & & & & & \\
\hline \multirow[t]{2}{*}{$4.50 E+0$} & -2 & 1.40 & +2 & -3 & 8.10 & 1.5 & 9.9 & 9.5 & & & & \\
\hline & & & & & & & & & & & & \\
\hline & & & & & & & & & & & & \\
\hline & 0 & 2.6 & 2.4 & 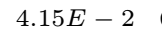 & 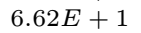 & $3.26 E+3$ & -1 & 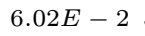 & & & & \\
\hline
\end{tabular}

Note: $\rho_{14}$ is the central density in units of $10^{14} \mathrm{~g} / \mathrm{cm}^{3} . M$ is the gravitational mass of the star. $R$ is the radius of the corresponding non-rotating configuration. $E_{\mathrm{B}}$ is the binding energy. $I_{44}$ is the moment of inertia (in units of $10^{44}$ C.G.S.). $\Omega_{c}\left(\mathrm{~s}^{-1}\right)$ is the critical angular velocity. $\omega_{1}$ is the dragging of inertial frames divided by the critical angular velocity. $\delta R / R$ is the relative correction induced by rotation in radius. $\delta M$ is the correction induced by rotation in gravitational mass. $e_{\mathrm{S}}$ is the radius at pole divided by the radius at equator. $Q / M R^{2}$ is the quadrupolar momentum. $\delta E_{\mathrm{B}}$ is the correction induced by rotation in binding energy.

rest-mass density in Fig. 5 (for $Y_{\mathrm{L}}=0.4$ and 0.3 ). In this figure we have plotted the equilibrium values for four isentropic configurations $(s=0.8,1.8,3.4,5)$. The maximum, for a given entropy, appears at central densities which are lower than the ones corresponding to $M_{\max }$. The moment of inertia is an increasing function of entropy; in particular, the maximum of the moment of inertia varies in a factor two when entropy varies from 0.8 to 3.4. The behaviour of the moment of inertia of hot protoneutron stars can be explained by taking into account that radius depends strongly on entropy. For the model of $1.4 M_{\odot}$, the moment of inertia (in units of $10^{44} \mathrm{~g} \mathrm{~cm}^{2}$ ) is $17 \leq I_{44} \leq 810$ when the entropy is $0.75 \leq s \leq 4.5$ for $Y_{\mathrm{L}}=0.3$ (see Tables 1 and 2). The curves of $I$ for a given value of entropy and two different $Y_{\mathrm{L}}$ have an intersection point at some value of the central rest-mass density lower than the corresponding maximum, with their values for $Y_{\mathrm{L}}=0.3$ lower than for $Y_{\mathrm{L}}=0.4$ at lower central densities. This is related to the behavior of the gravitational mass (Fig. 3, right).

The eccentricity evaluated on the surface, $e$, (see Appendix for definition) has been displayed as a function of the central rest-mass density in Fig. 6 (for $Y_{\mathrm{L}}=0.4$ and 0.3$)$ and for several values of the entropy $(s=0.8,1.8,3.4,5)$. It has a local maximum at low 
Table 2. Macroscopic properties of warm rotating neutron stars $\left(Y_{1}=0.4\right)$

\begin{tabular}{|c|c|c|c|c|c|c|c|c|c|c|c|c|}
\hline$S\left(K_{\mathrm{B}}\right)$ & $\rho_{14}$ & $M / M_{\odot}$ & $R(\mathrm{~km})$ & $E_{\mathrm{B}} / M_{\odot}$ & $I_{44}$ & $\Omega_{\mathrm{c}}\left(\mathrm{s}^{-1}\right)$ & $\omega_{1}$ & $\delta R / R$ & $\delta M / M$ & $e_{\mathrm{S}}$ & $Q / M R^{2}$ & $\delta E_{\mathrm{B}} / M_{\odot}$ \\
\hline \multirow[t]{3}{*}{$7.50 E-1$} & & & $3.76 E+1$ & $-2.84 E-3$ & $1.21 E+1$ & $E+2$ & & & $6 E-2$ & $6.85 E-1$ & $4.50 E-3$ & $1.29 E-4$ \\
\hline & $7.02 E+0$ & $1.40 E+0$ & $1.60 E+1$ & $6.89 E-2$ & $1.77 E+1$ & $4.54 E+3$ & $9.35 E-1$ & $5.77 E-2$ & $58 E-2$ & $6.71 E-1$ & $2.46 E-2$ & $9.36 E-3$ \\
\hline & $2.14 E+1$ & $1.92 E+0$ & $1.25 E+1$ & $1.84 E-1$ & $2.05 E+1$ & $7.59 E+3$ & $8.46 E-1$ & $3.47 E-2$ & $8.44 E-2$ & $6.94 E-1$ & $2.82 E-2$ & $1.14 E-2$ \\
\hline \multirow[t]{3}{*}{$1.00 E+0$} & $2.35 E+0$ & $5.62 E-1$ & $4.06 E+1$ & $-2.98 E-3$ & $1.33 E+1$ & $7.06 E+2$ & $9.97 E-1$ & $1.11 E-1$ & $2.86 E-2$ & $6.85 E-1$ & $3.56 E-3$ & $1.18 E-4$ \\
\hline & $6.81 E+0$ & $1.40 E+0$ & $1.64 E+1$ & $6.55 E-2$ & $1.80 E+1$ & $4.36 E+3$ & $9.39 E-1$ & $5.88 E-2$ & $9.22 E-2$ & $6.76 E-1$ & $2.30 E-2$ & $8.71 E-3$ \\
\hline & $2.14 E+1$ & $1.93 E+0$ & $1.27 E+1$ & $1.81 E-1$ & $2.08 E+1$ & $7.49 E+3$ & $8.49 E-1$ & $3.52 E-2$ & $8.27 E-2$ & $6.94 E-1$ & $2.72 E-2$ & $1.08 E-2$ \\
\hline \multirow[t]{3}{*}{$1.25 E+0$} & $2.26 E+0$ & $5.86 E-1$ & $4.15 E+1$ & $-3.16 E-3$ & $1.46 E+1$ & $6.98 E+2$ & $9.97 E-1$ & $1.11 E-1$ & $2.89 E-2$ & $6.85 E-1$ & $3.62 E-3$ & $1.24 E-4$ \\
\hline & $6.55 E+0$ & $1.40 E+0$ & $1.68 E+1$ & $6.12 E-2$ & $1.85 E+1$ & $4.19 E+3$ & $9.42 E-1$ & $5.99 E-2$ & $8.97 E-2$ & $6.76 E-1$ & $2.19 E-2$ & $8.12 E-3$ \\
\hline & $2.14 E+1$ & $1.94 E+0$ & $1.28 E+1$ & $1.76 E-1$ & $2.12 E+1$ & $7.39 E+3$ & $8.51 E-1$ & $3.55 E-2$ & $8.13 E-2$ & $6.94 E-1$ & $2.66 E-2$ & $1.03 E-2$ \\
\hline \multirow{3}{*}{$1.50 E+0$} & $2.09 E+0$ & $6.16 E-1$ & $4.39 E+1$ & $-3.36 E-3$ & $1.74 E+1$ & $6.56 E+2$ & $9.97 E-1$ & $1.11 E-1$ & $2.93 E-2$ & $6.85 E-1$ & $3.70 E-3$ & $1.27 E-4$ \\
\hline & $6.24 E+0$ & $1.40 E+0$ & $1.74 E+1$ & $5.61 E-2$ & $1.92 E+1$ & $3.98 E+3$ & $9.46 E-1$ & $6.13 E-2$ & $8.68 E-2$ & $6.76 E-1$ & $2.07 E-2$ & $7.42 E-3$ \\
\hline & $2.06 E+1$ & $1.96 E+0$ & $1.31 E+1$ & $1.71 E-1$ & $2.19 E+1$ & $7.19 E+3$ & $8.55 E-1$ & $3.63 E-2$ & $8.04 E-2$ & $6.94 E-1$ & $2.59 E-2$ & $1.01 E-2$ \\
\hline \multirow[t]{3}{*}{$1.75 E+0$} & $2.00 E+0$ & $6.52 E-1$ & $4.46 E+1$ & $-3.68 E-3$ & $1.92 E+1$ & $6.59 E+2$ & $9.97 E-1$ & $1.10 E-1$ & $2.96 E-2$ & $6.85 E-1$ & $3.76 E-3$ & $1.38 E-4$ \\
\hline & $5.88 E+0$ & $1.40 E+0$ & $1.81 E+1$ & $5.01 E-2$ & $2.00 E+1$ & $3.74 E+3$ & $9.50 E-1$ & $6.31 E-2$ & $8.32 E-2$ & $6.76 E-1$ & $1.92 E-2$ & $6.64 E-3$ \\
\hline & +1 & +0 & $1.34 E+1$ & $1.65 E-1$ & +1 & +3 & $E-1$ & $2 E-2$ & $7.92 E-2$ & $6.94 E-1$ & $2.50 E-2$ & $9.71 E-3$ \\
\hline $2.00 E+0$ & $1.93 E+0$ & $6.95 E-1$ & $4.54 E+1$ & $-4.04 E-3$ & $2.15 E+1$ & $6.64 E+2$ & $9.97 E-1$ & $1.10 E-1$ & $3.00 E-2$ & $6.85 E-1$ & $3.83 E-3$ & $1.53 E-4$ \\
\hline \multirow[t]{3}{*}{$2.25 E+0$} & $1.78 E+0$ & $7.44 E-1$ & $4.80 E+1$ & $-4.41 E-3$ & $2.61 E+1$ & $6.32 E+2$ & $9.96 E-1$ & $1.09 E-1$ & $3.06 E-2$ & $6.85 E-1$ & $3.96 E-3$ & $1.66 E-4$ \\
\hline & $5.07 E+0$ & $1.40 E+0$ & $2.02 E+1$ & $3.70 E-2$ & $2.24 E+1$ & $3.19 E+3$ & $9.60 E-1$ & $6.75 E-2$ & $7.47 E-2$ & $6.80 E-1$ & $1.60 E-2$ & $5.01 E-3$ \\
\hline & $1.83 E+1$ & $2.03 E+0$ & $1.42 E+1$ & $1.50 E-1$ & $2.50 E+1$ & $6.51 E+3$ & $8.69 E-1$ & $3.93 E-2$ & $7.61 E-2$ & $6.94 E-1$ & $2.31 E-2$ & $8.76 E-3$ \\
\hline \multirow{3}{*}{$2.50 E+0$} & $1.71 E+0$ & $8.03 E-1$ & $4.86 E+1$ & $-4.84 E-3$ & $2.96 E+1$ & $6.45 E+2$ & $E-1$ & $1.09 E-1$ & $3.12 E-2$ & $6.85 E-1$ & $4.08 E-3$ & $1.92 E-4$ \\
\hline & $4.59 E+0$ & $1.40 E+0$ & $2.16 E+1$ & $2.94 E-2$ & $2.39 E+1$ & +3 & -1 & $E-2$ & $E-2$ & $0 E-1$ & -2 & -3 \\
\hline & $E+1$ & $2.06 E+0$ & $1.46 E+1$ & $1.41 E-1$ & $2.64 E+1$ & +3 & $E-1$ & $E-2$ & $7.43 E-2$ & $6.99 E-1$ & -2 & -3 \\
\hline \multirow{3}{*}{$2.75 E+0$} & $1.58 E+0$ & $8.73 E-1$ & $5.07 E+1$ & $-5.19 E-3$ & $3.60 E+1$ & $6.29 E+2$ & $9.96 E-1$ & $1.08 E-1$ & $3.22 E-2$ & $6.85 E-1$ & $4.28 E-3$ & $E-4$ \\
\hline & $E+0$ & $1.40 E+0$ & $2.34 E+1$ & $2.24 E-2$ & $2.60 E+1$ & $2.55 E+3$ & $9.69 E-1$ & $7.38 E-2$ & $6.43 E-2$ & $6.85 E-1$ & -2 & $E-3$ \\
\hline & +1 & $2.09 E+0$ & +1 & $1.32 E-1$ & $2.79 E+1$ & $6.00 E+3$ & $8.80 E-1$ & $4.18 E-2$ & $7.21 E-2$ & $6.99 E-1$ & $2.09 E-2$ & $7.52 E-3$ \\
\hline \multirow{3}{*}{$3.00 E+0$} & $1.46 E+0$ & $9.53 E-1$ & $5.28 E+1$ & $-5.58 E-3$ & $4.40 E+1$ & $6.19 E+2$ & $9.96 E-1$ & $1.07 E-1$ & $3.34 E-2$ & $6.85 E-1$ & $4.52 E-3$ & $2.63 E-4$ \\
\hline & $3.62 E+0$ & $1.40 E+0$ & $2.57 E+1$ & $1.50 E-2$ & $2.88 E+1$ & $2.20 E+3$ & $9.75 E-1$ & $7.78 E-2$ & $5.86 E-2$ & $6.85 E-1$ & $1.07 E-2$ & $2.62 E-3$ \\
\hline & $1.62 E+1$ & $2.13 E+0$ & $1.57 E+1$ & $1.22 E-1$ & $2.97 E+1$ & $5.74 E+3$ & $8.85 E-1$ & $4.33 E-2$ & $6.99 E-2$ & $6.99 E-1$ & $1.97 E-2$ & $6.85 E-3$ \\
\hline \multirow[t]{3}{*}{$3.25 E+0$} & $1.35 E+0$ & $1.05 E+0$ & $5.46 E+1$ & $-5.85 E-3$ & $5.40 E+1$ & $6.17 E+2$ & -1 & 1.05 & $3.49 E-2$ & $6.85 E-1$ & $4.83 E-3$ & $3.19 E-4$ \\
\hline & $3.13 E+0$ & $1.40 E+0$ & $2.88 E+1$ & $8.55 E-3$ & $3.29 E+1$ & $1.87 E+3$ & $9 .^{\prime}$ & -2 & $5.30 E-2$ & $6.85 E-1$ & 9.09 & -3 \\
\hline & $1.50 E+1$ & $2.17 E+0$ & $1.64 E+1$ & $1.11 E-1$ & $3.21 E+1$ & $5.40 E+3$ & $8.92 E-1$ & $4.52 E-2$ & $6.78 E-2$ & $6.99 E-1$ & -2 & -3 \\
\hline \multirow{3}{*}{$3.50 E+0$} & $1.25 E+0$ & $1.16 E+0$ & $5.63 E+1$ & $-5.90 E-3$ & $6.66 E+1$ & $6.20 E+2$ & $9.94 E-1$ & $1.04 E-1$ & $3.67 E-2$ & $6.85 E-1$ & -3 & 4.03 \\
\hline & $E+0$ & $1.40 E+0$ & $3.30 E+1$ & $2.59 E-3$ & $3.89 E+1$ & $1.52 E+3$ & $9.84 E-1$ & $8.75 E-2$ & $4.75 E-2$ & $6.85 E-1$ & $7.65 E-3$ & -3 \\
\hline & $1.39 E+1$ & $2.22 E+0$ & $1.73 E+1$ & $9.95 E-2$ & $3.49 E+1$ & $5.05 E+3$ & $8.99 E-1$ & $4.74 E-2$ & $6.56 E-2$ & $6.99 E-1$ & $1.73 E-2$ & $5.92 E-3$ \\
\hline $3.75 E+0$ & $1.15 E+0$ & $1.29 E+0$ & $5.81 E+1$ & $-5.73 E-3$ & $8.32 E+1$ & $6.26 E+2$ & $9.94 E-1$ & $1.02 E-1$ & $3.88 E-2$ & $6.85 E-1$ & $5.69 E-3$ & $5.32 E-4$ \\
\hline $4.00 E+0$ & $1.18 E+1$ & $2.32 E+0$ & $1.93 E+1$ & $7.50 E-2$ & $4.19 E+1$ & $4.38 E+3$ & $9.13 E-1$ & $5.21 E-2$ & $6.07 E-2$ & $6.99 E-1$ & $1.47 E-2$ & $4.99 E-3$ \\
\hline \multirow[t]{2}{*}{$4.25 E+0$} & $7.47 E-1$ & $1.61 E+0$ & $6.80 E+1$ & $-4.66 E-3$ & $1.58 E+2$ & $5.51 E+2$ & $9.93 E-1$ & $9.83 E-2$ & $4.45 E-2$ & $6.85 E-1$ & $7.04 E-3$ & $8.32 E-4$ \\
\hline & & $2.38 E+0$ & $2.05 E+1$ & $6.19 E-2$ & $4.62 E+1$ & $4.05 E+3$ & $9.20 E-1$ & $5.47 E-2$ & $5.80 E-2$ & $6.99 E-1$ & $1.35 E-2$ & $4.48 E-3$ \\
\hline 4.5 & $1.01 E+1$ & $2.44 E+0$ & $2.18 E+1$ & $4.86 E-2$ & $5.12 E+1$ & $3.74 E+3$ & $9.27 E-1$ & $5.74 E-2$ & $5.52 E-2$ & $6.99 E-1$ & $1.23 E-2$ & $3.99 E-3$ \\
\hline+0 & $8.98 E+0$ & $2.51 E+0$ & $2.36 E+1$ & $3.52 E-2$ & $5.81 E+1$ & $3.37 E+3$ & $9.34 E-1$ & $6.07 E-2$ & $5.26 E-2$ & $6.99 E-1$ & $1.12 E-2$ & $3.69 E-3$ \\
\hline $5.00 E+0$ & $7.98 E+0$ & $2.59 E+0$ & $2.56 E+1$ & $2.17 E-2$ & $6.64 E+1$ & $3.03 E+3$ & $9.41 E-1$ & $6.42 E-2$ & $5.00 E-2$ & $6.94 E-1$ & $1.02 E-2$ & $3.39 E-3$ \\
\hline
\end{tabular}

Note: $\rho_{14}$ is the central density in units of $10^{14} \mathrm{~g} / \mathrm{cm}^{3} . M$ is the gravitational mass of the star. $R$ is the radius of the corresponding non-rotating configuration. $E_{\mathrm{B}}$ is the binding energy. $I_{44}$ is the moment of inertia (in units of $10^{44}$ C.G.S.). $\Omega_{\mathrm{c}}\left(\mathrm{s}^{-1}\right)$ is the critical angular velocity. $\omega_{1}$ is the dragging of inertial frames divided by the critical angular velocity. $\delta R / R$ is the relative correction induced by rotation in radius. $\delta M$ is the correction induced by rotation in gravitational mass. $e_{\mathrm{S}}$ is the radius at pole divided by the radius at equator. $Q / M R^{2}$ is the quadrupolar momentum. $\delta E_{\mathrm{B}}$ is the correction induced by rotation in binding energy.

central densities and intermediate masses. This local maxima of the function $e=e\left(\rho_{\mathrm{c}}, s\right)$ was already found by Chandrasekhar \& Miller (1974) in their study on slowly rotating homogeneous (energy density constant) masses and by Miller (1977) in his study of polytropic configurations. In Tables 1 and 2 we have shown the values of the quantity $e_{\mathrm{s}} \equiv r_{\mathrm{p}} / r_{\mathrm{e}}\left(r_{\mathrm{p}}\right.$ and $r_{\mathrm{e}}$ are, respectively, the values of the radial coordinate at pole and equator); hence, deviations of this quantity from one -the spherical valuegive an idea about the nonsphericity of the configuration. Tables 1 and 2 show that these models reach values as high as $\approx 34 \%$ of nonsphericity. For $1.4 M_{\odot} e_{\mathrm{s}}$ increases in the interval $0.66 \leq e_{\mathrm{s}} \leq 0.69$ when the entropy increases in the interval $0.75 \leq s \leq 4.0$ for $Y_{\mathrm{L}}=0.3$ (see Tables 1 and 2).

Deformations induced by rotation are, according to previous discussion, more relevant for intermediate masses than for the most massive objects allowed by the present EOS. Thermal effects decrease this tendency. Figure 7 shows, in a spatial two-dimensional diagram, the isopycnic curves (geometrical loci of points inside the star with the same value of the rest-mass density) of two models with the same baryonic mass $\left(1.4 M_{\odot}\right)$ and lepton abundance $\left(Y_{\mathrm{L}}=0.3\right)$. The characteristics of these models are indicated in the figure headings, which correspond to the values of entropy $s=0.75$ (left) and 4 (right). 

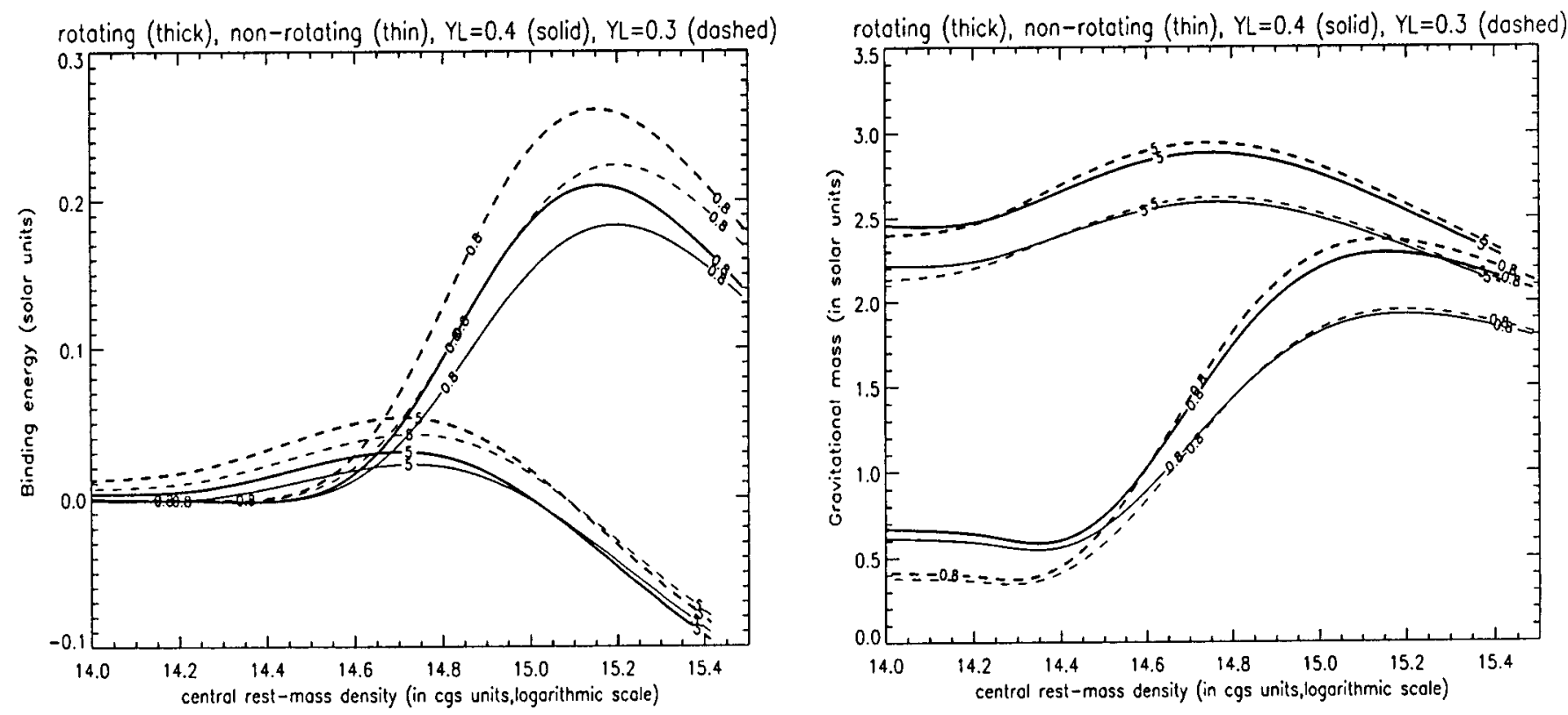

Fig. 3. Binding energy (left), in units of $M_{\odot} c^{2}$, and Gravitational mass (right), in solar units, as a function of the central restmass density (in cgs units and logarithmic scale) for two extreme values of entropy (labels stand for values $s=0.8$ and 5 , in units of the Boltzmann constant) for the non-rotating (thin) and rotating (thick) equilibrium configurations. Solid (dashed) lines correspond to $Y_{\mathrm{L}}=0.4\left(Y_{\mathrm{L}}=0.3\right)$

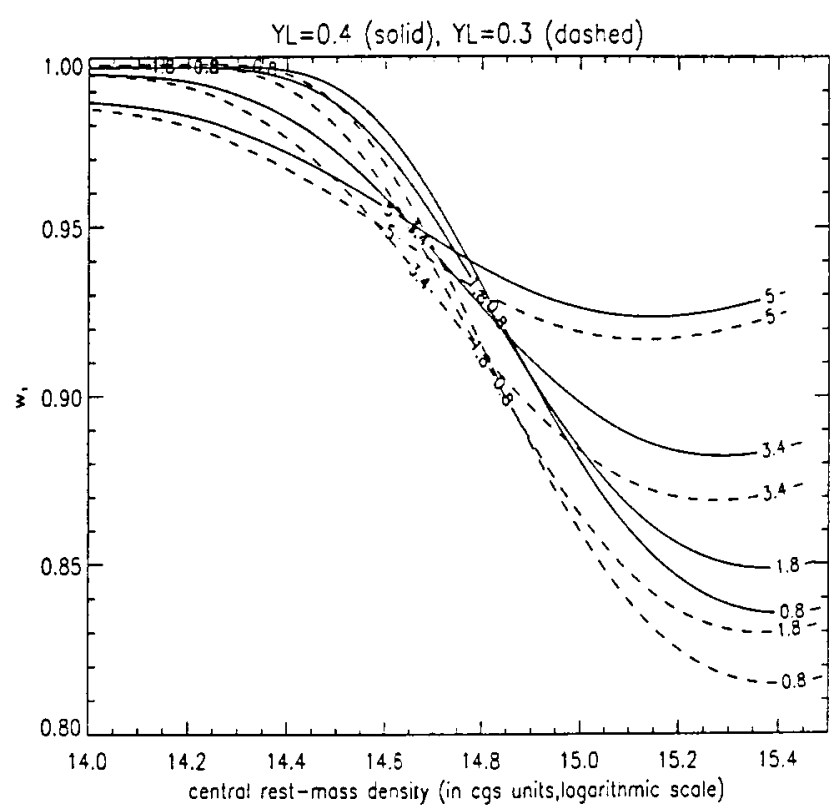

Fig. 4. Dragging of inertial frames evaluated at the surface. Quantity $w_{1}$ stands for $\frac{\bar{\omega}}{\Omega} \equiv 1-\frac{\omega}{\Omega}$ at $r=R$. It is plotted as a function of the central rest-mass density (in cgs units and logarithmic scale) for several values of entropy (labels stand for values $s=0.8,1.8,3.4$ and 5 , in units of the Boltzmann constant). Solid (dashed) lines correspond to $Y_{\mathrm{L}}=0.4\left(Y_{\mathrm{L}}=0.3\right)$

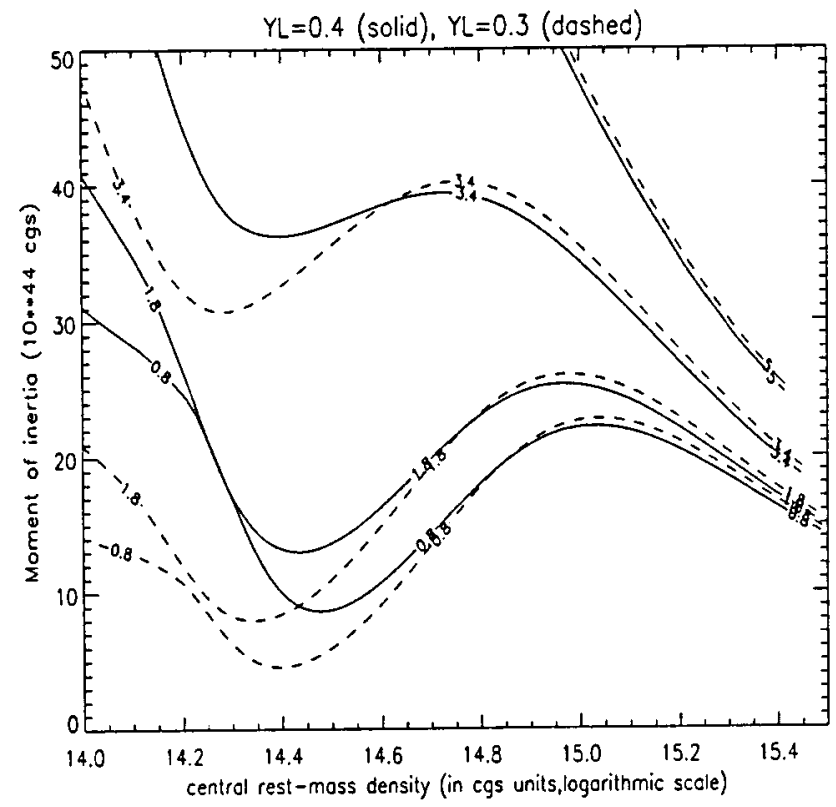

Fig. 5. Moment of inertia (in units of $10^{44} \mathrm{cgs}$ ) plotted as a function of the central rest-mass density (in cgs units and logarithmic scale) for several values of entropy (labels stand for values $s=0.8,1.8,3.4$ and 5 , in units of the Boltzmann constant). Solid (dashed) lines correspond to $Y_{\mathrm{L}}=0.4\left(Y_{\mathrm{L}}=0.3\right)$

For the sake of comparison, we have preferred to keep the same ratio for both axis (in km.) in both figures, and, therefore, the corresponding scales differ in a factor of three. We have also indicated (thick continuous line) the 


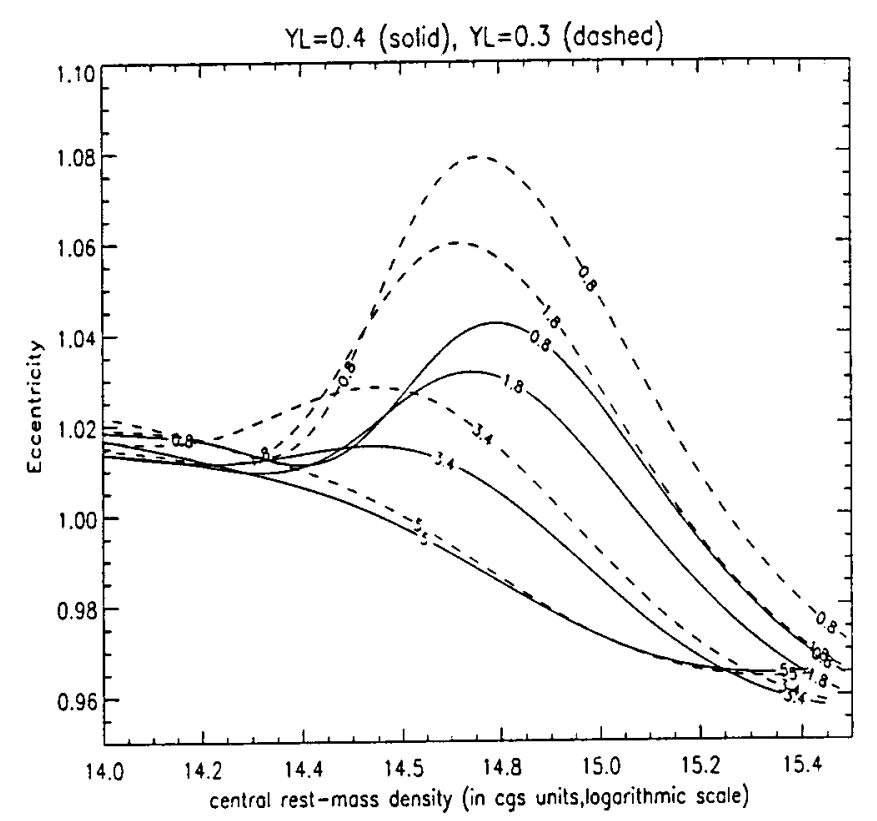

Fig. 6. Eccentricity, $\left[(\text { radius at equator })^{2} /(\text { radius at pole })^{2}-\right.$ $1]^{1 / 2}$, evaluated at the surface, plotted as a function of the central rest-mass density (in cgs units and logarithmic scale) for several values of entropy (labels stand for values $s=0.8,1.8$, 3.4 and 5 , in units of the Boltzmann constant). Solid (dashed) lines correspond to $Y_{\mathrm{L}}=0.4\left(Y_{\mathrm{L}}=0.3\right)$

surface of the corresponding spherical non-rotating model. Models shown in Fig. 7 have the critical angular velocity according to Eq. (1.1), with $C=C_{\mathrm{N}}$. As can be seen in Fig. 7 , the density gradient is sharper at the pole than at the equator. This may enhance the anisotropy of the flux of neutrinos emitted from the protoneutron star and may strengthen the deposit of energy for supernova explosion at polar direction (Janka \& Mönchmeyer 1989; Shimizu et al. 1994; Shimizu 1995). In a crude model according to which the neutrinos are radiated - assumed black body from a Maclaurin spheroid of semiaxis $r_{\mathrm{e}}$ and $r_{\mathrm{p}}$, the ratio between the local neutrino flux in the polar and equatorial directions, measured by some terrestrial detector, is $\frac{r_{\mathrm{e}}}{r_{\mathrm{p}}}$ (Shimizu 1995). In our case, for the models plotted in Fig. 7 , that ratio can be as high as $\approx 1.5$.

The star's mass quadrupole moment, $Q$ (see Appendix and Hartle 1967, for definition) measures the deformation of the star's exterior gravitational field. It is maximal for configurations of nearly uniform density and minimum for configurations with diffuse envelopes. When the central density increases the thermal effects become less important. As in the case of eccentricity, and for the model with $1.4 M_{\odot}$, quantity $Q / M R^{2}$ decreases in the interval $0.036 \geq Q / M R^{2} \geq 0.0085$ when entropy increases in the interval $0.75 \leq s \leq 4.0$ for $Y_{\mathrm{L}}=0.3$ (see Tables 1 and 2 ).

\section{Evolutionary analysis}

Nowadays, studying the evolution of a protoneutron star during its Kelvin epoch is a very active line of research, during which energy loss through neutrino emission dominates the star's outcome, and its consequence on the supernova neutrinos and the mechanism of supernova explosion. Sophisticated numerical codes have been built up in order to understand the physics involved in convection, neutrino transport and so on (see e.g., Burrows \& Lattimer 1986; Suzuki 1994; Janka \& Müller 1996; Keil et al. 1996). Realistic numerical simulations of the cooling of rotating protoneutron stars, including those relevant physical processes, are still in their infancy (see Janka \& Keil 1997). Therefore, in order to understand the generic features - structural and thermal changes - of the evolutionary history of rotating protoneutron stars, it might be of interest to follow the approach of just linking their stationary macroscopic properties corresponding to equilibrium configurations with different values of entropy per baryon and lepton fraction.

By collecting the data generated by our computations, discussed in the previous section, and reordering them we have generated a set of figures (Fig. 8), in which we have plotted - in a entropy versus central rest-mass density $(s-$ $\left.\rho_{\mathrm{c}}\right)$ diagram - the geometrical loci of those equilibrium configurations with the same baryonic mass (labels stand for this mass in solar units).

In Fig. 8, upper diagrams refer to non-rotating (i.e., zero angular momentum) protoneutron stars with $Y_{\mathrm{L}}=$ 0.3 (left) and $Y_{\mathrm{L}}=0.4$ (right). Lower diagrams in Fig. 8 display (for the same values of $Y_{\mathrm{L}}$ ) two families of protoneutron stars, parametrized by the baryonic mass: those non-rotating ones (continuous lines) and those having constant angular momentum (dashed lines). In this set of diagrams we have selected the values of the baryonic mass of a few ones. For the rotating configurations, and for the sake of simplicity (notice the existence of scaling laws), for each value of the baryonic mass, we have plotted those with maximum angular momentum. This maximum of the angular momentum is, in units of $10^{48} \mathrm{cgs}$ : i) 4.0, 9.5, 16.9, 25.0 and 30.5 for, respectively, the baryonic mass (in solar units) $0.9,1.4,1.9,2.4,2.63$ of the models shown in lower left diagram of Fig. $8\left(Y_{\mathrm{L}}=0.3\right)$, and ii) $3.5,8.5,15.5$, 24.0 and 25.7 for, respectively, the baryonic mass (in solar units) $0.9,1.4,1.9,2.4,2.5$ of the models shown in lower right diagram of Fig. $8\left(Y_{\mathrm{L}}=0.4\right)$.

The analysis of the diagrams in Fig. 8 can help one to understand the fundamental trends of the cooling of a protoneutron star, regarding to basic properties of the equilibrium configurations. First, we are going to make the assumption - which is quite obvious - that once the protoneutron star has formed, it starts to cool down keeping both its baryonic mass and its angular momentum constant- and, hence, the entropy (and the temperature at its inner core) decreases. Secondly, we are going to 

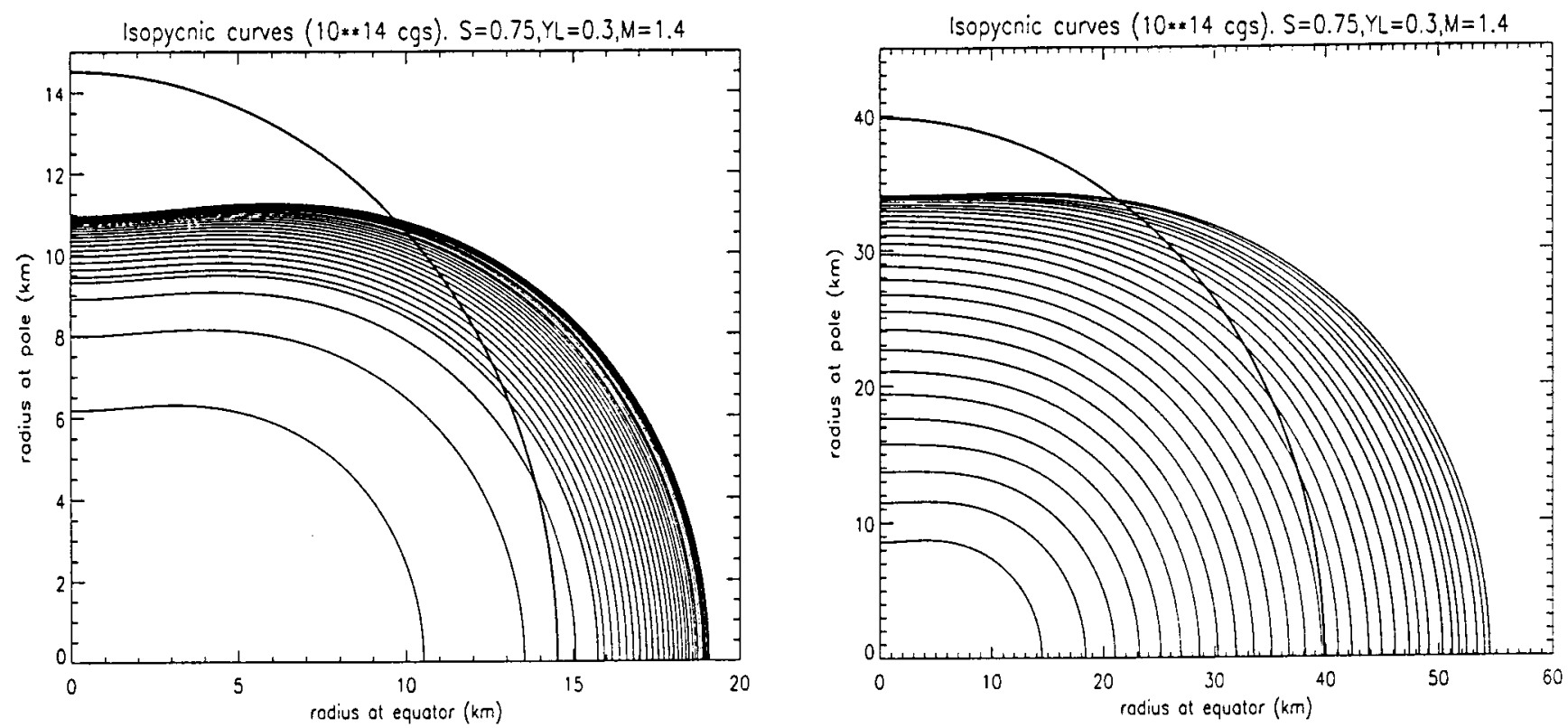

Fig. 7. Two-dimensional diagram displaying the isopycnic curves for the rotating model with $1.4 M_{\odot}$ (baryonic mass), entropy 0.75 (in units of the Boltzmann constant) and $Y_{\mathrm{L}}=0.3$. The model on the left has an angular velocity: $\Omega_{\mathrm{c}}=1.3910^{4} \mathrm{~s}^{-1}$. A total of 29 isocontours have been plotted spanning -at equispaced intervals in logarithmic scale- the total range of density, from the center $\left(\rho_{\mathrm{c}}=6.2010^{14} \mathrm{cgs}\right)$ to the surface $\left(\rho_{\mathrm{s}} \approx 510^{9} \mathrm{cgs}\right)$. The model on the right has an angular velocity: $\Omega_{\mathrm{c}}=6.9510^{3} \mathrm{~s}^{-1}$. A total of 29 isocontours have been plotted spanning - at equispaced intervals in logarithmic scale- the total range of density, from the center $\left(\rho_{\mathrm{c}}=1.5610^{14} \mathrm{cgs}\right)$ to the surface $\left(\rho_{\mathrm{s}} \approx 510^{9} \mathrm{cgs}\right)$. For the sake of comparison we have also added the curve showing the position of the surface of the corresponding non-rotating model

ignore the short interval of time in which the unbounced inner core heats due to thermal waves (propagating inwards from the region where the shock was formed) as well as the outward diffusion of electron neutrinos produced by electron captures onto protons in the inner core. Guided by these two basic principles, the information contained in diagrams of Fig. 8 illustrates the following issues.

i) For a given value of $Y_{\mathrm{L}}$, a critical value of the baryonic mass exists, $M_{\mathrm{A}}^{\mathrm{c}}$ (the maximum for the cold configurations for the same $Y_{\mathrm{L}}$ ) such that stars with a mass $M_{\mathrm{A}}>M_{\mathrm{A}}^{\mathrm{c}}$ will become unstable and will evolve towards a black hole, increasing in central density and entropy. From Fig. 8 (see also Tables 1 and 2) we can find some of the values of $M_{\mathrm{A}}^{\mathrm{c}}$ : they are, roughly, $\approx 2.1 M_{\odot}\left(\approx 2.5 M_{\odot}\right)$ for non-rotating (rotating) configurations, with negligible differences due to $Y_{\mathrm{L}}$.

ii) The value of $M_{\mathrm{A}}^{\mathrm{c}}$ of the rotating configurations (for a given $Y_{\mathrm{L}}$ ) is an upper bound for the non-rotating ones. This statement seems obvious, but let us draw the reader's attention towards the curve labelled by 2.5 in lower right diagram of Fig. 8. There are no stable protoneutron stars with $M_{\mathrm{A}}>M_{\mathrm{A}}^{\mathrm{c}} \approx 2.5$ (for $Y_{\mathrm{L}}=0.4$ ). If they were born with that baryonic mass it would not be possible for them to cool down into a stable neutron star for both rotating and non-rotating cases.

iii) Only allowing the value of $Y_{\mathrm{L}}$ to decrease during cooling (that is what should happen when neutrinos leak out of the star, according to standard theory) it is possible to relax the upper bound quoted in ii) a bit. A rotating protoneutron star of $2.5 M_{\odot}$ - according to this picture will cool down to lower and lower values of entropy eventually reaching a zero value for $Y_{\mathrm{L}}=0.3$ or the bifurcation point to the unstable branch for $Y_{\mathrm{L}}=0.4$.

iv) For a given value of the baryonic mass and the entropy, the central rest-mass density of equilibrium configurations decreases with the angular momentum.

v) Finally, let us point out that during cooling, rotating protoneutron stars undergo a significant increase of their rest-mass density at the center. This feature displayed in Fig. 8 together with the above issues can be of potential interest if phase transitions in dense matter exist (as already pointed out in Martí et al. 1988).

\section{Summary and conclusions}

We have studied the properties of general relativistic slowly rotating protoneutron stars. We have surveyed the structure of rotating protoneutron stars with a wide range of the entropy per baryon, the lepton fraction and the baryonic mass of the stars in order to shed light on the evolutionary history of protoneutron stars during cooling after birth. We have adopted the relativistic equation of state of dense matter derived by the relativistic mean field theory, which is based on the microscopic nuclear manybody framework and checked by the experimental data of many nuclei. 

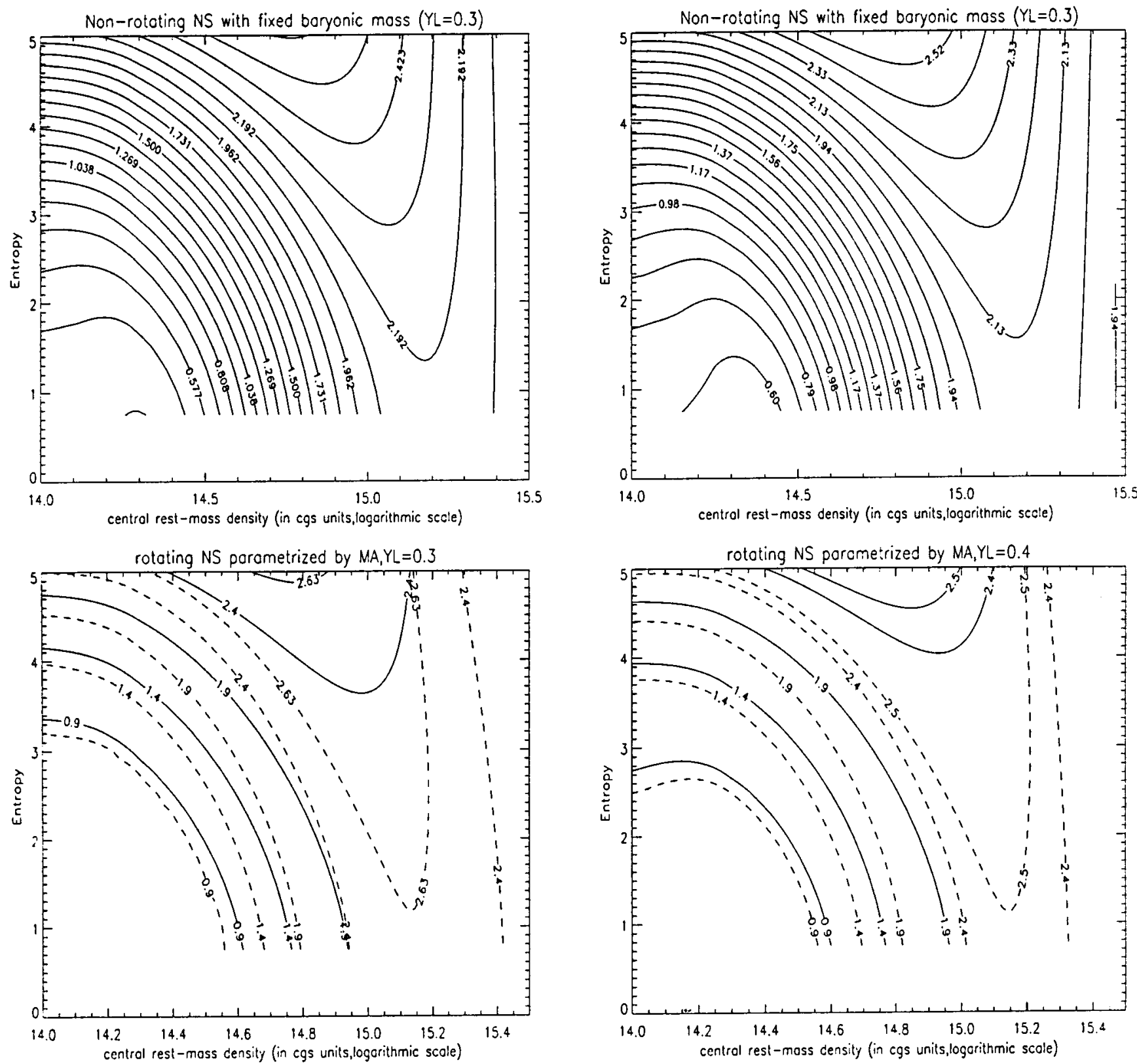

Fig. 8. Entropy (in units of the Boltzmann constant) versus central rest-mass density (in cgs units and logarithmic scale) diagrams. The diagrams on the upper panel show the curves which are the geometrical loci of those non-rotating equilibrium configurations having the same baryonic mass (labels stand for the mass, in solar units) and lepton abundance $Y_{\mathrm{L}}=0.3$ (left) and $Y_{\mathrm{L}}=0.4$ (right). The diagrams on the lower panel show the curves which are the geometrical loci of those non-rotating (continuous lines) and rotating (dashed lines), with the maximum angular momentum, equilibrium configurations having the same baryonic mass (labels stand for the mass, in solar units) and lepton abundance $Y_{\mathrm{L}}=0.3$ (left) and $Y_{\mathrm{L}}=0.4$ (right)

We have presented the macroscopic properties of warm rotating protoneutron stars in the tables and the figures, so as to provide basic information on the effect of rotation and general relativity on protoneutron stars with various baryonic masses for a wide variety of thermal conditions. The information enables us to construct the evolutionary history for cooling and deleptonizing protoneutron stars by following the change of thermal states $\left(s\right.$ and $\left.Y_{\mathrm{L}}\right)$ depending on baryonic masses.
The masses and radii of protoneutron stars are affected by rotation in up to $10 \%$. The effects of rotation become weaker for stars with larger entropy per baryon. This is due to the fact that larger radii of the stars with larger entropy per baryon lead to smaller critical angular velocities. Macroscopic properties of stars depend rather weakly on the lepton fraction but depend more significantly on the entropy per baryon. 
The shapes of rotating protoneutron stars are deformed with a value of about 0.7 for $e_{\mathrm{s}}$. The gradients of density at equator and pole are found to be different. This leads to the anisotropy of neutrino flux and may have an influence on the observational neutrino events and the mechanism of delayed supernova explosion. During the cooling epoch, both the shape of stars and the density gradient change according to the corresponding entropy per baryon and lepton fraction and the neutrino flux may vary accordingly.

Following the history of protoneutron stars having the same baryonic mass, the central density is found to increase during the cooling and this may lead to a possible phase transition of dense matter inside during the cooling of protoneutron stars. The analysis reveals that, for a given value of $Y_{\mathrm{L}}$, a critical value of the baryonic mass exists, which is the maximum of the cold configurations for the same $Y_{\mathrm{L}}$. For rotating protoneutron stars, the critical mass is about $2.5 M_{\odot}$, above which protoneutron stars will evolve towards a black hole.

Acknowledgements. This work has been supported by the Spanish DGICYT (grant PB94-0973). K.S. is grateful for fruitful discussions with members of Universidad de Valencia, where the most of this work has been done, and their warm hospitality. K.S. thanks also for the Alexander von Humboldt Stiftung, which partly supports his visit to Valencia during his research stay in Max-Planck-Institute für Astrophysik.

\section{Appendix A: Hartle's formalism}

We have solved the equations derived by Hartle and Thorne which describe the structure of slowly rotating relativistic stars (Hartle 1967; Hartle \& Thorne 1968). When the static equilibrium configuration is perturbed by rotation, the geometry of space-time is changed. For an appropriate choice of coordinates, the perturbed geometry is described by $(\mathrm{c}=\mathrm{G}=1)$

$$
\begin{aligned}
\mathrm{d} s^{2}= & -\mathrm{e}^{\nu}\left[1+2\left(h_{0}+h_{2} P_{2}\right)\right] \mathrm{d} t^{2} \\
& +\frac{\left[1+2\left(m_{0}+m_{2} P_{2}\right) /(r-2 m)\right]}{1-2 m / r} \mathrm{~d} r^{2} \\
& +r^{2}\left[1+2\left(v_{2}-h_{2}\right) P_{2}\right]\left[\mathrm{d} \theta^{2}\right. \\
& \left.+\sin ^{2} \theta(\mathrm{d} \varphi-\omega \mathrm{d} t)^{2}\right]+O\left(\Omega^{3}\right),
\end{aligned}
$$

where $P_{2}$ is the Legendre polynomial of order $2 ; \omega$, which is called the angular velocity of the local inertial frame, is a function of $r$ and is proportional to the star's angular velocity $\Omega$. In the above equations $h_{0}, h_{2}, m_{0}, m_{2}$ and $v_{2}$ are all functions of $r$ proportional to $\Omega^{2}$. The quantity $\bar{\omega} \equiv \Omega-\omega$ is the angular velocity of the fluid relative to the local inertial frame.

The surface of constant density in the rotating configuration is given by the radius

$r+\xi_{0}(r)+\xi_{2}(r) P_{2}(\cos \theta)$,

where

$$
\xi_{0}=-p_{0}^{*}(E+p) /(\mathrm{d} p / \mathrm{d} r),
$$

(in fact, this equation defines $p_{0}^{*}$ )

$\xi_{2}=-p_{2}^{*}(E+p) /(\mathrm{d} p / \mathrm{d} r)$,

$p_{2}^{*}=-h_{2}-\frac{r^{2} \bar{\omega}^{2}}{3 \mathrm{e}^{\nu}}$.

In order to have an invariant parametrization of a surface of constant density, it can be embedded in a threedimensional flat space, with coordinates $r^{*}, \theta^{*}$ and $\phi^{*}$. The surface in the flat space is, to order $\Omega^{2}$, the spheroid $r^{*}\left(\theta^{*}\right)=r+\xi_{0}(r)+$

$$
\left\{\xi_{2}(r)+r\left[v_{2}(r)-h_{2}(r)\right]\right\} P_{2}\left(\cos \theta^{*}\right) .
$$

The mean radius of this spheroid is

$\bar{r}^{*}=r+\xi_{0}(r)$

After some algebraic manipulations, Einstein equations for a perfect fluid can be written as:

$\frac{\mathrm{d} m}{\mathrm{~d} r}=4 \pi r^{2} \rho$

$\frac{\mathrm{d} p}{\mathrm{~d} r}=-\frac{(\rho+p)\left(m+4 \pi r^{3} p\right)}{r(r-2 m)}$

$\frac{\mathrm{d} \nu}{\mathrm{d} r}=2 \frac{m+4 \pi r^{3} p}{r(r-2 m)}$

$\frac{\mathrm{d} \xi}{\mathrm{d} r}=\frac{u}{r^{4}}-\frac{4 \pi r^{4}(\rho+p)}{r-2 m}$

$\frac{\mathrm{d} u}{\mathrm{~d} r}=\frac{16 \pi r^{5} \xi(\rho+p)}{r-2 m}$,

$\frac{\mathrm{d} m_{0}}{\mathrm{~d} r}=4 \pi r^{2}(\rho+p) \frac{\mathrm{d} \rho}{\mathrm{d} p} p_{0}^{*}+\frac{u^{2}}{12 r^{4}}+\frac{8 \pi r^{5}(\rho+p) \xi^{2}}{3(r-2 m)}$

$$
\begin{aligned}
\frac{\mathrm{d} p_{0}^{*}}{\mathrm{~d} r}= & \frac{u^{2}}{12 R^{4}(r-2 m)}-\frac{m_{0}\left(1+8 \pi r^{2} p\right)}{(r-2 m)^{2}} \\
& -\frac{4 \pi(\rho+p) r^{2} p_{0}^{*}}{r-2 m}+\frac{2 \xi r^{2}}{3(r-2 m)}\left[\frac{u}{r^{3}}\right. \\
& \left.+\frac{\left(r-3 m-4 \pi r^{3} p\right) \xi}{r-2 m}\right]
\end{aligned}
$$

$$
\begin{aligned}
\frac{\mathrm{d}\left(\delta E_{\mathrm{B}}\right)}{4 \pi r^{2} \mathrm{~d} r}= & (\rho+p) p_{0}^{*}\left\{\frac{\mathrm{d} \rho}{\mathrm{d} p}\left[\left(1-\frac{2 m}{r}\right)^{-1 / 2}-1\right]\right. \\
& \left.-\left(\frac{\mathrm{d} \rho}{\mathrm{d} p}-\frac{\mathrm{d} \rho_{0}}{\mathrm{~d} p}\right)\left(1-\frac{2 m}{r}\right)^{-1 / 2}\right\} \\
& +\rho_{0}\left(1-\frac{2 m}{r}\right)^{-3 / 2}\left[\frac{m_{0}}{r}+\frac{r^{2} \xi^{2}}{3}\right] \\
& -\frac{1}{4 \pi r^{2}}\left[\frac{u^{2}}{12 r^{4}}+\frac{8 \pi r^{5}(\rho+p) \xi^{2}}{3 r(r-2 m)}\right]
\end{aligned}
$$

$$
\begin{aligned}
\frac{\mathrm{d} v_{2}}{\mathrm{~d} r}= & \frac{1}{r(r-2 m)}\left\{-2 h_{2}\left(m+4 \pi r^{3} p\right)+(r-m+\right. \\
& \left.\left.4 \pi r^{3} p\right)\left[\frac{8 \pi r^{5}(\rho+p) \xi^{2}}{3(r-2 m)}+\frac{u^{2}}{6 r^{4}}\right]\right\},
\end{aligned}
$$




$$
\begin{aligned}
\frac{\mathrm{d} h_{2}}{\mathrm{~d} r}= & h_{2}\left\{\frac{r^{2}}{2\left(m+4 \pi r^{3} p\right)}\left[8 \pi(\rho+p)-\frac{4 m}{r^{3}}\right]\right. \\
& \left.-\frac{2\left(m+4 \pi r^{3} p\right)}{r(r-2 m)}\right\}-\frac{2 v_{2}}{m+4 \pi r^{3} p} \\
& +\frac{u^{2}}{6 r^{4}}\left[\frac{m+4 \pi r^{3} p}{r(r-2 m)}-\frac{1}{2\left(m+4 \pi r^{3} p\right)}\right] \\
& +\frac{8 \pi r^{5}(\rho+p) \xi^{2}}{3(r-2 m)}\left[\frac{m+4 \pi r^{3} p}{r(r-2 m)}\right. \\
& \left.+\frac{1}{2\left(m+4 \pi r^{3} p\right)}\right] .
\end{aligned}
$$

We have introduced the intermediate variables $u$ and $\xi$ in order to split the equation of second order for $\bar{\omega}$ into a system of two equations of first order:

$u=r^{4} j \frac{\mathrm{d} \bar{\omega}}{\mathrm{d} r}$,

where

$j=\mathrm{e}^{-\nu / 2} \sqrt{1-\frac{2 m}{r}}$,

$\xi=j \bar{\omega}$.

We must also integrate the homogeneous equations for $h_{2}$ and $v_{2}$ in order to obtain the general solution

$\frac{\mathrm{d} v_{2}^{\mathrm{h}}}{\mathrm{d} r}=-2 \mathrm{~h}_{2}^{\mathrm{h}} \frac{m+4 \pi r^{3} p}{r(r-2 m)}$,

$$
\begin{aligned}
\frac{\mathrm{d} h_{2}^{\mathrm{h}}}{\mathrm{d} r}= & h_{2}^{\mathrm{h}}\left\{\frac{r^{2}}{2\left(m+4 \pi r^{3} p\right)}\left[8 \pi(\rho+p)-\frac{4 m}{r^{3}}\right]\right. \\
& \left.-\frac{2\left(m+4 \pi r^{3} p\right)}{r(r-2 m)}\right\}-\frac{2 v_{2}^{\mathrm{h}}}{m+4 \pi r^{3} p} .
\end{aligned}
$$

The expression for $\frac{\mathrm{d} \rho}{\mathrm{d} P}$ can be deduced from the relativistic thermal equilibrium condition and the equation of hydrostatic equilibrium

$$
\left(\frac{\mathrm{d} \rho}{\mathrm{d} p}\right)_{\mathrm{H.E.}}=\left(\frac{\partial p}{\partial \rho}\right)_{\mathrm{T}}^{-1}\left[1-T(\rho+p)^{-1}\left(\frac{\partial p}{\partial T}\right)_{\rho}\right] .
$$

The boundary conditions at the center are the following:

$$
\begin{array}{ll}
\nu_{\mathrm{c}}=0, & \left(\frac{\mathrm{d} \nu}{\mathrm{d} r}\right)_{\mathrm{c}}=0, \\
\xi_{\mathrm{c}}=j_{\mathrm{c}} \bar{\omega}_{\mathrm{c}}=\bar{\omega}=1, & \left(\frac{\mathrm{d} \xi}{\mathrm{d} r}\right)_{\mathrm{c}}=0, \\
u_{\mathrm{c}}=0, & \left(\frac{\mathrm{d} u}{\mathrm{~d} r}\right)_{\mathrm{c}}=0, \\
m_{0 \mathrm{c}}=0, & \left(\frac{\mathrm{d} m_{0}}{\mathrm{~d} r}\right)_{\mathrm{c}}=0, \\
p_{0 \mathrm{c}}^{*}=0, & \left(\frac{\mathrm{d} p_{0 \mathrm{c}}^{*}}{\mathrm{~d} r}\right)_{\mathrm{c}}=0, \\
\delta E_{\mathrm{B}_{\mathrm{c}}}=0, & \left(\frac{\mathrm{d} \delta E_{\mathrm{B}}}{\mathrm{d} r}\right)_{\mathrm{c}}=0, \\
v_{2 \mathrm{c}}=0, & \left(\frac{\mathrm{d} v_{2}}{\mathrm{~d} r}\right)_{\mathrm{c}}=0,
\end{array}
$$

$$
\begin{array}{ll}
h_{2 \mathrm{c}}=0, & \left(\frac{\mathrm{d} h_{2}}{\mathrm{~d} r}\right)_{\mathrm{c}}=0, \\
v_{2 \mathrm{c}}^{\mathrm{h}}=0, & \left(\frac{\mathrm{d} v_{2}^{\mathrm{h}}}{\mathrm{d} r}\right)_{\mathrm{c}}=0, \\
h_{2 \mathrm{c}}^{\mathrm{h}}=0, & \left(\frac{\mathrm{d} h_{2}^{\mathrm{h}}}{\mathrm{d} r}\right)_{\mathrm{c}}=0 .
\end{array}
$$

The homogeneous equations for $h_{2}$ and $v_{2}$ must verify the following conditions at the center

$\lim _{r \rightarrow 0} h_{2}^{\mathrm{h}}=A r^{2}$,

$\lim _{r \rightarrow 0} v_{2}^{\mathrm{h}}=B r^{4}$

with $A$ and $B$ non null constants related by

$B+2 \pi\left(p_{c}+\frac{p}{3}\right) A=0$.

The general solution can be written

$v_{2}=A^{\prime} v_{2}^{\mathrm{h}}+v_{2}^{\mathrm{p}}$,

$h_{2}=A^{\prime} h_{2}^{\mathrm{h}}+h_{2}^{\mathrm{p}}$.

Outside the star $h_{2}$ and $v_{2}$ have the analytic form:

$h_{2}=J^{2}\left(\frac{1}{M r^{3}}+\frac{1}{r^{4}}\right)+K Q_{2}^{2}\left(\frac{r}{M}-1\right)$,
$v_{2}=-\frac{J^{2}}{r^{4}}+\frac{2 K M}{[r(r-2 M)]^{1 / 2}} Q_{2}^{1}\left(\frac{r}{M}-1\right)$,

where $K$ is a constant, $Q_{n}^{m}$ is the associated Legendre polynomial of the second kind and $J$ is given by:

$J=\frac{u e^{-\nu / 2}}{6 \sqrt{1-2 \frac{M}{R}}}$.

$K$ and $A^{\prime}$ are obtained by matching $h_{2}$ and $v_{2}$ to the external solution. But before doing this we must scale all the variables by the appropriate factor so that the potential at the surface and $\bar{\omega}$ reach the correct values given by the expressions:

$\nu_{\mathrm{S}}=2 \frac{\log \left(\sqrt{1-\frac{2 M}{R}}\right)}{2}$,

$\Omega_{\mathrm{k}}=\bar{\omega}+\frac{2 J}{R^{3}}=\sqrt{\frac{M}{R^{3}}}$.

We have integrated the above system of equations with the corresponding boundary conditions from the center to the surface by using standard ODE solvers (fourth order Runge-Kutta). The star's surface is fixed by the condition $P(r=R)=P\left(\rho_{\text {surf }}\right), \rho_{\text {surf }} \approx 510^{9} \mathrm{~g} / \mathrm{cm}^{3}$.

The correction to the gravitational mass induced by rotation is then

$\delta M=m_{0}(R)+J^{2} / R^{3}$.

We have calculated the "amu" mass $\left(M_{\mathrm{A}}\right)$ and the "proper" mass $\left(M_{\mathrm{P}}\right)$ :

$M_{\mathrm{A}}=4 \pi \int_{0}^{R} r^{2} \mathrm{e}^{\Lambda} \rho_{0} \mathrm{~d} r$ 
$M_{\mathrm{P}}=4 \pi \int_{0}^{R} r^{2} \mathrm{e}^{\Lambda} \rho \mathrm{d} r$

with

$\mathrm{e}^{\Lambda}=\left(1-\frac{2 m}{r}\right)^{-1 / 2}$.

The mean adiabatic index is given by

$\left\langle\Gamma_{1}\right\rangle=\frac{\int_{0}^{R} r^{2} p(1-2 m / r)^{-1 / 2} \Gamma_{1} \mathrm{~d} r}{\int_{0}^{R} r^{2} p(1-2 m / r)^{-1 / 2} \mathrm{~d} r}$.

Other quantities of interest are: the moment of inertia

$I=\frac{J}{\Omega}$

the binding energy

$E_{\mathrm{B}}=M_{\mathrm{A}}-M$,

the change in the binding energy due to the rotation is

$\delta E_{\mathrm{B}}=\delta M_{\mathrm{A}}-\delta M$.

The total number of baryons, $A$, in the nonrotating star is related to $M_{\mathrm{A}}$ by

$M_{\mathrm{A}}=\mu A$,

where $\mu$ is the rest mass per baryon.

The quadrupolar momentum is defined as

$Q=\frac{8 K M^{3}}{5}+\frac{J^{2}}{M}$.

Finally, the eccentricity at the surface, $e$, of the spheroid is defined by

$e=\left[(\text { radius at equator })^{2} /(\text { radius at pole })^{2}-1\right]^{1 / 2}$

$=\left[-3\left(v_{2}-h_{2}+\xi_{0} / r\right)\right]^{1 / 2}$.

Let us notice the fact that quantities such as $\delta M, \delta R$ $\left(\equiv \xi_{0}(R)\right), \delta E_{\mathrm{B}}$ and $Q$ scale as $\Omega^{2}$ and $e$ does as $\Omega$.

\section{References}

Baldo M., Bombaci I., Burgio G.F., 1997, A\&A 328, 274

Bombaci I., Prakash M., Prakash M., Ellis P.J., Lattimer J.M., Brown G.E., 1995, Nucl. Phys. A583, 623

Bombaci I., 1996, A\&A 305, 871

Bombaci I., Prakash M., Prakash M., Ellis P.J., Lattimer J.M., Brown G.E., 1997, Phys. Reports 280, 1

Brockmann R., Machleidt R., 1990, Phys. Rev. C42, 1965

Burrows A., Lattimer J.M., 1986, ApJ 307, 178

Burrows A., 1990, in Supernovae, Petschek A.G. (ed.). Springer, p. 143

Canal R., Isern J., Labay J., 1990, Ann. Rev. Astron. Astrophys. 28, 183
Chandrasekhar S., Miller J.C., 1974, MNRAS 167, 63

Datta B., 1988, Fund. Cosmic Phys. 12, 151

Diaz Alonso J., Ibáñez J.Mํ.., Sivak H., 1989, Phys. Rev. C39 671

Friedman J.L., Ipser J.R., Parker L., 1986, ApJ 304, 115

Friedman J.L., Ipser J.R., Parker L., 1989, Phys. Rev. Lett. 62,3015

Glendenning, 1997, Compact Objects. Springer

Goussard J.O., 1997, Ph.D. Thesis, Université de Paris VII

Goussard J.O., Haensel P., Zdunik J.L., 1997, A\&A 321, 822

Haensel P., Zdunik J.L., 1989, Nat 340, 617

Hartle J.B., 1967, ApJ 150, 1005

Hartle J.B., Thorne K.S., 1968, ApJ 153, 807

Hashimoto M., Oyamatsu K., Eriguchi Y., 1994, ApJ 436, 257

Hirata D., Toki H., Watabe T., Tanihata I., Carlson B.V., 1991, Phys. Rev. C44, 1467

Hirata D., Sumiyoshi K., Tanihata I., Sugahara Y., Tachibana T., Toki H., 1997, Nucl. Phys. A616, 438

Janka H.-T., Keil W., 1997, in Supernovae and Cosmology, Proc. of the Colloquium in Honor of Prof. G. Tamman (astro-ph/9709012)

Janka H.-T., Mönchmeyer R., 1989, A\&A 209, L5

Janka H.-T., Müller E., 1996, A\&A 306, 167

Kapoor R.C., Datta B., 1985, ApJ 297, 413

Kapoor R.C., Datta B., 1986, ApJ 311, 680

Keil W., Janka H.-T., Müller E., 1996, ApJ 473, L111

Lattimer J.M., Swesty F.D., 1991, Nucl. Phys. A535, 331

Martí J.M., Miralles J.A., Diaz Alonso J., Ibáñez J.Mạ., 1988, ApJ 329, 780

Miller J.C., 1977, MNRAS 179, 483

Romero J.V., Diaz Alonso J., Ibáñez J.Mํㅡ., Miralles J.A., Pérez A., 1992, ApJ 395, 612

Serot B.D., Walecka J.D., 1986, Adv. Nucl. Phys. 16, 1

Shimizu T.M., Yamada S., Sato K., 1994, ApJ 432, L119

Shimizu T.M., 1995, Ph.D. Thesis. University of Tokyo

Sugahara Y., Toki H., 1994, Nucl. Phys. A579, 557

Sugahara Y., 1995, Ph.D. Thesis. Tokyo Metropolitan University

Sumiyoshi K., Toki H., 1994, ApJ 422, 700

Sumiyoshi K., Kuwabara H., Toki H., 1995, Nucl. Phys. A581, 725

Sumiyoshi K., Oyamatsu K., Toki H., 1995, Nucl. Phys. A595, 327

Sumiyoshi K., Suzuki H., Toki H., 1995, A\&A 303, 475

Suzuki H., 1994, in: "Physics and Astrophysics of Neutrinos", Fukugita M., Suzuki A. (eds.). Springer-Verlag, Tokyo, p. 763; and references therein

Takatsuka T., 1995, Nucl. Phys. A588, 365c

Weaver T.A., Woosley S.E., 1993, Phys. Reports 227, 65

Woosley S.E., Langer N., Weaver T.A., 1993, ApJ 411, 823 